\title{
Analysis of HIV models with multiple target cell populations and general nonlinear rates of viral infection and cell death
}

\author{
Xia Wang ${ }^{\mathrm{a}, \mathrm{b}, *}$, Xinyu Song ${ }^{\mathrm{b}}$, Sanyi Tang ${ }^{\mathrm{a}}$, Libin Rong ${ }^{\mathrm{c}}$ \\ ${ }^{a}$ School of Mathematics and Information Sciences, Shaanxi Normal University, \\ Xi'an, 710062, China \\ ${ }^{\mathrm{b}}$ College of Mathematics and Information Science, Xinyang Normal University, \\ Xinyang, 464000, China \\ ${ }^{\mathrm{c}}$ Department of Mathematics and Statistics, and Center for Biomedical Research, \\ Oakland University, Rochester, MI 48309
}

\begin{abstract}
HIV can infect different cell populations such as CD4+ T cells and macrophages. In this paper, we study the global property of the solution of an HIV model with two target cell populations. The model includes general nonlinear rates of viral infection and cell death. For each class of target cells, the time delay between viral entry into cells and viral production is included in the model. We obtain the basic reproductive number of the model, which is shown to provide a threshold condition determining the long-term behavior of the solution of the model. Specifically, we show that the infection-free equilibrium is globally asymptotically stable when the basic reproductive number is less than or equal to 1 , and that the infected equilibrium is globally asymptotically stable when the basic reproductive number is greater than 1 . We also extend the model with two target cell populations to a general model with $n$ populations. Similar global properties are obtained for the general model. Numerical simulations are performed to illustrate the stability results and to evaluate the relative contribution to viral production from the two cell populations.
\end{abstract}

Key words: Virus dynamics; Delay; Multiple target cells; Global stability; Lyapunov functional.

AMS subject classification: 34C05, 92D25

* Corresponding author. E-mail address: xywangxia@163.com (X. Wang). 


\section{Introduction}

It is well known that mathematical modeling and analysis plays an important role in the study of epidemiology $[1,2,15,17,20,25]$. For example, Anderson and May constructed epidemic models embodying the essentials of the interaction between invertebrate hosts and their directly transmitted microparasites to study the dynamical behavior of conventional epidemiology [2]. Mathematical models have also been developed to study within-host dynamics of HIV infection, i.e., the interaction between cells, virus, and immune responses [4,22-24,26,31,36,39,40,42,50]. In [26], McLean and Kirkwood developed a mathematical model of the activation and proliferation of a clone of $\mathrm{T}$ helper cells in response to a replicating antigen and investigated the circumstance under which HIV can destabilize persistent infection and destroy immune memory. Rong and Perelson [39] reviewed mathematical models used to study HIV dynamics under antiretroviral therapy, low viral load persistence, the stability of latently infected cells, and the emergence of transient viral load measurements above the detection limit (so-called "viral blips"). A basic viral dynamic model of HIV infection is given below.

$$
\left\{\begin{array}{l}
\dot{x}(t)=\lambda-d x(t)-\beta x(t) v(t), \\
\dot{y}(t)=\beta x(t) v(t)-a y(t) \\
\dot{v}(t)=p y(t)-\delta v(t)
\end{array}\right.
$$

where $x, y$ and $v$ represent the densities of uninfected target cells, infected cells and virus, respectively, in blood at time $t$. Uninfected cells are assumed to be generated at a constant rate $\lambda$, die at a per capita rate $d$, and become infected at a rate $\beta x v$, where $\beta$ is the infection rate. Infected cells are produced at a rate $\beta x v$ and die at a per capita rate $a$. Virus is assumed to be produced by productively infected cells at a rate $p$ per cell, and is removed from blood at a per capita rate $\delta$.

The mass action term $\beta x v$ is used in model (1) to describe viral infection. However, the infection may not be strictly linear in each variable over the entire range of virions $v$ and target cells $x$. Nonlinear contact rate has been used in some mathematical models $[9,17,18,48]$. For example, Wang et al. [48] used a virus infection rate with the Beddington-DeAngelis functional response $x v /(1+k x+c v)$. Georgescu and Hsieh [9] considered a nonlinear infection rate with $c(x) f(v)$, where $c(x)$ is the contact rate function depending on susceptible cells $x$ and $f(v)$ represents the force of infection by virus at density $v$. Huang et al. [14] and Korobeinikov $[17,18]$ assumed the incidence rate to be a nonlinear function $\varphi(x, v)$. In addition, there exists a time delay between viral entry into a target cell and viral production. This intracellular time delay has been studied in many models $[6,10,12,14,21,27-30,46,47,49]$. In this paper, we will include a time delay $\tau$ in the general incidence of viral infection, i.e. $\varphi(x(t-\tau), v(t-\tau))$.

The death rate of productively infected cells may not follow the exponential decay described by $-a y(t)$ in model (1). Productively infected cells are mainly cleared by the immune response, which is stimulated by infected cells [5]. Thus, the death rate 
of productively infected cells may depend on the density of infected cells [39]. Holte et al. [13] assumed the death rate to be $a(y)=a y^{\omega}$, where $\omega$ governs the effect of the immune response on the death rate. In this paper, we will use a general nonlinear function $a G(y)$ to describe the death rate of productively infected cells.

All of the above-mentioned HIV infection models study the interaction between HIV and $\mathrm{CD} 4^{+} \mathrm{T}$ cells. In addition to $\mathrm{CD} 4^{+} \mathrm{T}$ cells, other cells such as macrophages [16] and dendritic cells [35] are known to be susceptible to HIV infection. HIV can infect macrophages through binding of gp120 to CD4 and CCR5 receptors, and were identified to be a highly productive source of HIV during the latter stage of viral infection [32]. Two-compartment models [5,34,39] are needed to study the interaction between HIV and two target cell populations. Elaiw and his collaborators have investigated the global asymptotic stability of the steady states of HIV models with two classes of target cells $[7,8,45]$.

In the present paper, we study the global property of the solution of an HIV model with two target cell populations. The model includes general nonlinear rates of viral infection and cell death. Time delays between viral entry into each population of target cells and viral production are incorporated into the model. We analyze the model by deriving the basic reproductive number and proving the global stability of steady states. We also extend the model by including $n$ target cell populations. Similar global properties are obtained for the general model and numerical simulations are performed to illustrate the theoretical results.

\section{A model with two target cell populations}

In this section, we introduce the following mathematical model of HIV infection with two time delays and two classes of target cells, $\mathrm{CD} 4^{+} \mathrm{T}$ cells and macrophages.

$$
\left\{\begin{array}{l}
\dot{x}_{1}(t)=\lambda_{1}-d_{1} x_{1}(t)-\varphi_{1}\left(x_{1}(t), v(t)\right), \\
\dot{x}_{2}(t)=\lambda_{2}-d_{2} x_{2}(t)-\varphi_{2}\left(x_{2}(t), v(t)\right), \\
\dot{y}_{1}(t)=e^{-\mu_{1} \tau_{1}} \varphi_{1}\left(x_{1}\left(t-\tau_{1}\right), v\left(t-\tau_{1}\right)\right)-a_{1} G_{1}\left(y_{1}\right), \\
\dot{y}_{2}(t)=e^{-\mu_{2} \tau_{2}} \varphi_{2}\left(x_{2}\left(t-\tau_{2}\right), v\left(t-\tau_{2}\right)\right)-a_{2} G_{2}\left(y_{2}\right), \\
\dot{v}(t)=p_{1} G_{1}\left(y_{1}(t)\right)+p_{2} G_{2}\left(y_{2}(t)\right)-\delta v(t) .
\end{array}\right.
$$

In the model, $x_{1}(t)$ and $x_{2}(t)$ are the populations of two classes of uninfected target cells, CD4 ${ }^{+} \mathrm{T}$ cells and macrophages, respectively. $y_{1}(t)$ and $y_{2}(t)$ represent the populations of of productively infected cells that can produce virions. $\lambda_{1}$ and $\lambda_{2}$ are the rates at which target cells are generated, $d_{1}$ and $d_{2}$ are their death rates, and the functions $\varphi_{1}\left(x_{1}, v\right), \varphi_{2}\left(x_{2}, v\right)$ are the rates at which two target cell populations are infected by virus. For each population, a time delay $\left(\tau_{1}\right.$ or $\left.\tau_{2}\right)$ is incorporated to describe the time between initial viral entry and viral production. $e^{-\mu_{1} \tau_{1}}$ (or $e^{-\mu_{2} \tau_{2}}$ ) is the probability of infected $\mathrm{CD} 4^{+} \mathrm{T}$ cells (or macrophages) surviving to 
produce virions, where $\mu_{1}$ (or $\left.\mu_{2}\right)$ is a constant death rate of infected CD4 ${ }^{+} \mathrm{T}$ cells (or macrophages) that have not began to produce virions.

As addressed in the Introduction, we use nonlinear functions $a_{1} G\left(y_{1}\right)$ and $a_{2} G\left(y_{2}\right)$ to represent the death rates of infected cells, where $a_{1}, a_{2}$ are positive constants. We assume that an infected cell releases the same number of virions when it dies. Thus, the viral production rate is proportional to the death rate $\left(a_{1} G\left(y_{1}\right)\right.$ or $\left.a_{2} G\left(y_{2}\right)\right)$ of infected cells, i.e., the viral production rates are $p_{1} G_{1}\left(y_{1}\right)$ and $p_{2} G_{2}\left(y_{2}\right)$, respectively, for the two populations. Virus is assumed to be cleared at a rate $\delta$.

\subsection{Preliminaries}

We assume that the functions $\varphi_{i}\left(x_{i}, v\right)$ and $G_{i}\left(y_{i}\right)$ in system (2) are always positive, differentiable, and monotonically increasing for all $x_{i}>0, y_{i}>0$ and $v>0$. We also assume that $\varphi_{i}\left(x_{i}, v\right)$ is concave downward with respect to $v, i=1,2$. That is, they satisfy the following assumptions:

$\left(\mathrm{H}_{1}\right) \varphi_{i}(0, v)=\varphi\left(x_{i}, 0\right)=0, \frac{\partial \varphi_{i}\left(x_{i}, 0\right)}{\partial v}>0$ for $x_{i}>0$ and $v>0, G_{i}(0)=$ $0, G_{i}{ }^{\prime}\left(y_{i}\right)>0$ for $y_{i}>0, i=1,2$.

$\left(\mathrm{H}_{2}\right) \varphi_{i}\left(x_{i}, v\right)>0, \frac{\partial \varphi_{i}\left(x_{i}, v\right)}{\partial x_{i}}>0, \frac{\partial \varphi_{i}\left(x_{i}, v\right)}{\partial v}>0$ for any $x_{i}>0$ and $v>0, i=1,2$.

$\left(\mathrm{H}_{3}\right) \varphi_{i}\left(x_{i}, v\right)$ is concave downward with respect to $v$. This ensures that

$$
\varphi_{i}\left(x_{i}, v\right) \leq v \frac{\partial \varphi_{i}\left(x_{i}, 0\right)}{\partial v} .
$$

The initial condition of system (2) is given by

$$
x_{1}(\theta)=\psi_{1}(\theta), x_{2}(\theta)=\psi_{2}(\theta), y_{1}(\theta)=\psi_{3}(\theta), y_{2}(\theta)=\psi_{4}(\theta), v(\theta)=\psi_{5}(\theta) .
$$

Here $\psi=\left(\psi_{1}, \psi_{2}, \psi_{3}, \psi_{4}, \psi_{5}\right)^{T} \in C\left([-\tau, 0], R^{5}\right)$, which is the Banach space of continuous functions from $[-\tau, 0]$ to $R^{5}$ equipped with the following sup-norm

$$
\left\|\psi_{j}\right\|=\sup _{\theta \in[-\tau, 0]} \psi_{j}(\theta), \quad \tau=\max \left\{\tau_{1}, \tau_{2}\right\}, \quad j=1,2, \cdots, 5 .
$$

By the fundamental theory of functional differential equations [19], any solution $\left(x_{1}(t), x_{2}(t), y_{1}(t), y_{2}(t), v(t)\right)^{T}$ of system $(2)$ exists and is differentiable for all $t>0$. Furthermore, the state space $X=C^{+}=C\left([-\tau, 0], R_{+}^{5}\right)$ is positively invariant for system (2).

Lemma 2.1 Under the above initial conditions (3), $x_{1}(t), y_{1}(t), x_{2}(t), y_{2}(t)$ and $v(t)$ are all nonnegative and bounded in $X$.

Proof. A similar argument as in the proof of Theorem 1 in [14] can show that any solution of system (2) with initial conditions (3) are nonnegative and bounded in $X$. 
According to Lemma 2.1, we know that the following bounded feasible region

$$
\begin{gathered}
\Omega=\left\{\left(x_{1}, y_{1}, x_{2}, y_{2}, v\right) \in C^{+}:\left\|x_{i}\right\| \leq x_{i}^{0},\left\|y_{i}\right\| \leq M,\|v\| \leq M,\right. \\
M>0 \text { is a constant }\} \subseteq X
\end{gathered}
$$

is positively invariant with respect to system (2).

\subsection{Equilibria and basic reproduction number}

System (2) has an infection-free equilibrium point $E^{0}=\left(x_{1}^{0}, x_{2}^{0}, 0,0,0\right)$, where $x_{i}^{0}=$ $\lambda_{i} / d_{i}, i=1,2$. If an infected equilibrium point $E^{*}=\left(x_{1}^{*}, x_{2}^{*}, y_{1}^{*}, y_{2}^{*}, v^{*}\right)$ exists, it must satisfy $x_{i}^{*}, y_{i}^{*}, v^{*}>0$ for $i=1,2$, and

$$
\left\{\begin{array}{l}
0=\lambda_{1}-d_{1} x_{1}^{*}-\varphi_{1}\left(x_{1}^{*}, v^{*}\right), \\
0=\lambda_{2}-d_{2} x_{2}^{*}-\varphi_{2}\left(x_{2}^{*}, v^{*}\right), \\
0=e^{-\mu_{1} \tau_{1}} \varphi_{1}\left(x_{1}^{*}, v^{*}\right)-a_{1} G_{1}\left(y_{1}^{*}\right), \\
0=e^{-\mu_{2} \tau_{2}} \varphi_{2}\left(x_{2}^{*}, v^{*}\right)-a_{2} G_{2}\left(y_{2}^{*}\right), \\
0=p_{1} G_{1}\left(y_{1}^{*}\right)+p_{2} G_{2}\left(y_{2}^{*}\right)-\delta v^{*}
\end{array}\right.
$$

We can show that system (2) is uniformly persistent by a similar argument as in the proof of Proposition 3.3 in ref. [20]. Together with the boundedness of the solution in the interior of the set $\Omega$, denoted by int $\Omega$, we know that there exists a positive equilibrium point $E^{*}$ of system (2) in int $\Omega$.

The dynamics of the virus infection model (2) depend on the basic reproduction number $R_{0}$. Using the next generation method for ordinary differential equation systems in van den Driessche and Watmough [43] and for delay differential equation systems (see $[14,21]$ ), the basic reproduction number of our system is obtained as follows:

$$
R_{0}=\sum_{i=1}^{2} \frac{p_{i}}{a_{i} \delta} \cdot \frac{\partial \varphi_{i}\left(x_{i}^{0}, 0\right)}{\partial v} e^{-\mu_{i} \tau_{i}}
$$

where $x_{i}^{0}=\lambda_{i} / d_{i}, i=1,2$. The term $p_{i} / a_{i}$ represents the number of virions produced by one virus-producing cell. $1 / \delta$ is the lifespan of a virion. The term $\frac{\partial \varphi_{i}\left(x_{i}^{0}, 0\right)}{\partial v}$ denotes the maximal number of target cells that one virion can infect per unit time, and $e^{-\mu_{i} \tau_{i}}$ represents the probability of infected cells surviving to produce new virions. If there is only one population of target cells, then $R_{0}$ in (5) becomes the basic reproduction number in ref. [14]. 


\section{Main results}

In this section, we will construct Lyapunov functionals to study the global dynamics of system (2). We need the following assumptions for the nonlinear functions $\varphi(x, v)$ and $G(y)$.

$\left(\mathrm{H}_{4}\right) \frac{\partial \varphi_{i}\left(x_{i}, 0\right)}{\partial v}$ is increasing with respect to $x_{i}>0, i=1,2$.

$\left(\mathrm{H}_{5}\right) x_{i}-x_{i}^{0}-\int_{x_{i}^{0}}^{x_{i}} \lim _{v \rightarrow 0^{+}} \frac{\varphi_{i}\left(x_{i}^{0}, v\right)}{\varphi_{i}(\sigma, v)} d \sigma \rightarrow+\infty$ as $x_{i} \rightarrow 0^{+}$;

$$
\begin{aligned}
& x_{i}-x_{i}^{*}-\int_{x_{i}^{*}}^{x_{i}} \frac{\varphi_{i}\left(x_{i}^{*}, v^{*}\right)}{\varphi_{i}\left(\sigma, v^{*}\right)} d \sigma \rightarrow+\infty \text { as } x_{i} \rightarrow 0^{+} \text {or } x_{i} \rightarrow+\infty ; \\
& y_{i}-y_{i}^{*}-\int_{x_{i}^{*}}^{y_{i}} \frac{G_{i}\left(y_{i}^{*}\right)}{G_{i}(\sigma)} d \sigma \rightarrow+\infty \text { as } y_{i} \rightarrow 0^{+} \text {or } y_{i} \rightarrow+\infty .
\end{aligned}
$$

Theorem 3.1 Suppose that conditions $\left(\mathrm{H}_{1}\right)-\left(\mathrm{H}_{5}\right)$ are satisfied. If $R_{0} \leq 1$, then the infection-free equilibrium $E^{0}$ of system (2) is globally asymptotically stable.

Proof. Define a Lyapunov functional

$$
L_{1}(t)=W_{1}\left(x_{1 t}, x_{2 t}, y_{1 t}, y_{2 t}, v_{t}\right)+W_{+},
$$

where

$$
\begin{aligned}
W_{1}= & e^{-\mu_{1} \tau_{1}}\left[x_{1}(t)-x_{1}^{0}-\int_{x_{1}^{0}}^{x_{1}(t)} \lim _{v \rightarrow 0^{+}} \frac{\varphi_{1}\left(x_{1}^{0}, v\right)}{\varphi_{1}(\sigma, v)} d \sigma\right]+y_{1}(t) \\
& +\alpha e^{-\mu_{2} \tau_{2}}\left[x_{2}(t)-x_{2}^{0}-\int_{x_{2}^{0}}^{x_{2}(t)} \lim _{v \rightarrow 0^{+}} \frac{\varphi_{2}\left(x_{2}^{0}, v\right)}{\varphi_{2}(\sigma, v)} d \sigma\right]+\alpha y_{2}(t)+\frac{a_{1}}{p_{1}} v(t), \\
W_{+}= & e^{-\mu_{1} \tau_{1}} \int_{0}^{\tau_{1}} \varphi_{1}\left(x_{1}(t-\theta), v(t-\theta)\right) d \theta+\alpha e^{-\mu_{2} \tau_{2}} \int_{0}^{\tau_{2}} \varphi_{2}\left(x_{2}(t-\theta), v(t-\theta)\right) d \theta,
\end{aligned}
$$

where $\alpha=\frac{a_{1} p_{2}}{a_{1} p_{1}}$.

Calculating the time derivative of $W_{1}$ along the solution of system (2), we obtain

$$
\begin{aligned}
\left.\dot{W}_{1}\right|_{(2)}= & e^{-\mu_{1} \tau_{1}}\left(1-\lim _{v \rightarrow 0^{+}} \frac{\varphi_{1}\left(x_{1}^{0}, v\right)}{\varphi_{1}\left(x_{1}, v\right)}\right)\left(\lambda_{1}-d_{1} x_{1}-\varphi_{1}\left(x_{1}, v\right)\right) \\
& +e^{-\mu_{1} \tau_{1}} \varphi_{1}\left(x_{1}\left(t-\tau_{1}\right), v\left(t-\tau_{1}\right)\right)-a_{1} G_{1}\left(y_{1}\right) \\
& +\alpha e^{-\mu_{2} \tau_{2}}\left(1-\lim _{v \rightarrow 0^{+}} \frac{\varphi_{2}\left(x_{2}^{0}, v\right)}{\varphi_{2}\left(x_{2}, v\right)}\right)\left(\lambda_{2}-d_{2} x_{2}-\varphi_{2}\left(x_{2}, v\right)\right) \\
& +\alpha\left(e^{-\mu_{2} \tau_{2}} \varphi_{2}\left(x_{2}\left(t-\tau_{2}\right), v\left(t-\tau_{2}\right)\right)-a_{2} G_{2}\left(y_{2}\right)\right) \\
& +\frac{a_{1}}{p_{1}}\left(p_{1} G_{1}\left(y_{1}(t)\right)+p_{2} G_{2}\left(y_{2}(t)\right)-\delta v(t)\right) .
\end{aligned}
$$


Similarly,

$$
\begin{aligned}
\left.\dot{W}_{+}\right|_{(2)}= & \frac{d}{d t}\left[e^{-\mu_{1} \tau_{1}} \int_{0}^{\tau_{1}} \varphi_{1}\left(x_{1}(t-\theta), v(t-\theta)\right) d \theta\right. \\
& \left.+\alpha e^{-\mu_{2} \tau_{2}} \int_{0}^{\tau_{2}} \varphi_{2}\left(x_{2}(t-\theta), v(t-\theta)\right) d \theta\right] \\
= & e^{-\mu_{1} \tau_{1}} \int_{0}^{\tau_{1}} \frac{d}{d t} \varphi_{1}\left(x_{1}(t-\theta), v(t-\theta)\right) d \theta \\
& +\alpha e^{-\mu_{2} \tau_{2}} \int_{0}^{\tau_{2}} \frac{d}{d t} \varphi_{2}\left(x_{2}(t-\theta), v(t-\theta)\right) d \theta \\
= & -e^{-\mu_{1} \tau_{1}} \int_{0}^{\tau_{1}} \frac{d}{d \theta} \varphi_{1}\left(x_{1}(t-\theta), v(t-\theta)\right) d \theta \\
& -\alpha e^{-\mu_{2} \tau_{2}} \int_{0}^{\tau_{2}} \frac{d}{d \theta} \varphi_{2}\left(x_{2}(t-\theta), v(t-\theta)\right) d \theta \\
= & e^{-\mu_{1} \tau_{1}}\left(\varphi_{1}\left(x_{1}, v\right)-\varphi_{1}\left(x_{1}\left(t-\tau_{1}\right), v\left(t-\tau_{1}\right)\right)\right) \\
& +\alpha e^{-\mu_{2} \tau_{2}}\left(\varphi_{2}\left(x_{2}, v\right)-\varphi_{2}\left(x_{2}\left(t-\tau_{2}\right), v\left(t-\tau_{2}\right)\right)\right) .
\end{aligned}
$$

Using $\lambda_{i}=d_{i} x_{i}^{0}$ and $\alpha=a_{1} p_{2} /\left(a_{2} p_{1}\right)$, from (7)-(8) we have

$$
\begin{aligned}
\left.\dot{L}_{1}(t)\right|_{(2)}= & \dot{W}_{1}+\dot{W}_{+} \\
= & e^{-\mu_{1} \tau_{1}} d_{1} x_{1}(t)\left(\frac{x_{1}^{0}}{x_{1}(t)}-1\right)\left(1-\lim _{v \rightarrow 0^{+}} \frac{\varphi_{1}\left(x_{1}^{0}, v\right)}{\varphi_{1}\left(x_{1}, v\right)}\right) \\
& +\alpha e^{-\mu_{2} \tau_{2}} d_{2} x_{2}(t)\left(\frac{x_{2}^{0}}{x_{2}(t)}-1\right)\left(1-\lim _{v \rightarrow 0^{+}} \frac{\varphi_{2}\left(x_{2}^{0}, v\right)}{\varphi_{2}\left(x_{2}, v\right)}\right) \\
& +e^{-\mu_{1} \tau_{1}} \varphi_{1}\left(x_{1}, v\right) \lim _{v \rightarrow 0^{+}} \frac{\varphi_{1}\left(x_{1}^{0}, v\right)}{\varphi_{1}\left(x_{1}, v\right)} \\
& +\alpha e^{-\mu_{2} \tau_{2}} \varphi_{2}\left(x_{2}, v\right) \lim _{v \rightarrow 0^{+}} \frac{\varphi_{2}\left(x_{2}^{0}, v\right)}{\varphi_{2}\left(x_{2}, v\right)}-\frac{\delta a_{1}}{p_{1}} v(t) \\
= & e^{-\mu_{1} \tau_{1}} d_{1} x_{1}(t)\left(\frac{x_{1}^{0}}{x_{1}(t)}-1\right)\left(1-\lim _{v \rightarrow 0^{+}} \frac{\varphi_{1}\left(x_{1}^{0}, v\right)}{\varphi_{1}\left(x_{1}, v\right)}\right) \\
& +\alpha e^{-\mu_{2} \tau_{2}} d_{2} x_{2}(t)\left(\frac{x_{2}^{0}}{x_{2}(t)}-1\right)\left(1-\lim _{v \rightarrow 0^{+}} \frac{\varphi_{2}\left(x_{2}^{0}, v\right)}{\varphi_{2}\left(x_{2}, v\right)}\right) \\
& +\frac{\delta a_{1}}{p_{1}} v(t)\left[\frac{p_{1} e^{-\mu_{1} \tau_{1}}}{\delta a_{1}} \frac{\varphi_{1}\left(x_{1}, v\right)}{v} \lim _{v \rightarrow 0^{+}} \frac{\varphi_{1}\left(x_{1}^{0}, v\right)}{\varphi_{1}\left(x_{1}, v\right)}\right. \\
& \left.+\frac{\alpha p_{1} e^{-\mu_{2} \tau_{2}}}{\delta a_{1}} \frac{\varphi_{2}\left(x_{2}, v\right)}{v} \lim _{v \rightarrow 0^{+}} \frac{\varphi_{2}\left(x_{2}^{0}, v\right)}{\varphi_{2}\left(x_{2}, v\right)}-1\right]
\end{aligned}
$$

The condition $\left(\mathrm{H}_{4}\right)$ is equivalent to

$$
\left(\frac{x_{i}^{0}}{x_{i}(t)}-1\right)\left(1-\frac{\frac{\partial \varphi_{i}\left(x_{i}^{0}, 0\right)}{\partial v}}{\frac{\partial \varphi_{i}\left(x_{i}, 0\right)}{\partial v}}\right) \leq 0, \quad i=1,2 .
$$

Therefore,

$$
\left(\frac{x_{i}^{0}}{x_{i}(t)}-1\right)\left(1-\lim _{v \rightarrow 0^{+}} \frac{\varphi_{i}\left(x_{i}^{0}, v\right)}{\varphi_{i}\left(x_{i}, v\right)}\right)=\left(\frac{x_{i}^{0}}{x_{i}(t)}-1\right)\left(1-\frac{\frac{\partial \varphi_{i}\left(x_{i}^{0}, 0\right)}{\partial v}}{\frac{\partial \varphi_{i}\left(x_{i}, 0\right)}{\partial v}}\right) \leq 0
$$


Furthermore, by the concavity of $\varphi_{i}\left(x_{i}, v\right)$ with respect to $v$ in $\left(\mathrm{H}_{3}\right)$, we have

$$
\varphi_{i}\left(x_{i}, v\right) \leq v \frac{\partial \varphi_{i}\left(x_{i}, 0\right)}{\partial v}
$$

Thus,

$$
\begin{aligned}
& \frac{p_{1} e^{-\mu_{1} \tau_{1}}}{\delta a_{1}} \frac{\varphi_{1}\left(x_{1}, v\right)}{v} \lim _{v \rightarrow 0^{+}} \frac{\varphi_{1}\left(x_{1}^{0}, v\right)}{\varphi_{1}\left(x_{1}, v\right)}+\frac{\alpha p_{1} e^{-\mu_{2} \tau_{2}}}{\delta a_{1}} \frac{\varphi_{2}\left(x_{2}, v\right)}{v} \lim _{v \rightarrow 0^{+}} \frac{\varphi_{2}\left(x_{2}^{0}, v\right)}{\varphi_{2}\left(x_{2}, v\right)}-1 \\
& =\sum_{i=1}^{2} \frac{p_{i}}{\delta a_{i}} \cdot e^{-\mu_{i} \tau_{i}} \cdot \frac{\varphi_{i}\left(x_{i}, v\right)}{v} \cdot \frac{\frac{\partial \varphi_{i}\left(x_{i}^{0}, 0\right)}{\partial v}}{\frac{\partial \varphi_{i}\left(x_{i}, 0\right)}{\partial v}}-1 \\
& \leq \sum_{i=1}^{2} \frac{p_{i}}{\delta a_{i}} \cdot e^{-\mu_{i} \tau_{i}} \cdot \frac{\partial \varphi_{i}\left(x_{i}^{0}, 0\right)}{\partial v}-1=R_{0}-1 .
\end{aligned}
$$

From (9), (11) and (13), we obtain

$$
\begin{aligned}
\left.\dot{L}_{1}(t)\right|_{(2)} \leq & e^{-\mu_{1} \tau_{1}} d_{1} x_{1}(t)\left(\frac{x_{1}^{0}}{x_{1}(t)}-1\right)\left(1-\lim _{v \rightarrow 0^{+}} \frac{\varphi_{1}\left(x_{1}^{0}, v\right)}{\varphi_{1}\left(x_{1}, v\right)}\right) \\
& +\alpha e^{-\mu_{2} \tau_{2}} d_{2} x_{2}(t)\left(\frac{x_{2}^{0}}{x_{2}(t)}-1\right)\left(1-\lim _{v \rightarrow 0^{+}} \frac{\varphi_{2}\left(x_{2}^{0}, v\right)}{\varphi_{2}\left(x_{2}, v\right)}\right) \\
& +\frac{\delta a_{1}}{p_{1}} v(t)\left(R_{0}-1\right) \\
\leq & \frac{\delta a_{1}}{p_{1}} v(t)\left(R_{0}-1\right),
\end{aligned}
$$

and $\left.\dot{L}_{1}\right|_{(2)}=0$ if and only if $v(t) \equiv 0$ or $R_{0} \equiv 1$ and $x=x_{i}^{0}$. Therefore, the maximal compact invariant set in $\left\{\left.\dot{L}_{1}\right|_{(2)}=0\right\}$ is the singleton $\left\{E^{0}\right\}$. By LaSalle Invariance Principle for delay differential equations (see $[11,44]), E^{0}$ is globally attractive. It can be further shown that $E^{0}$ is locally stable using the similar method as in Corollary 5.3.1 of ref. [11]. Therefore, $E^{0}$ is globally asymptotically stable in $X$.

Theorem 3.2 Suppose that conditions $\left(\mathrm{H}_{1}\right)-\left(\mathrm{H}_{5}\right)$ are satisfied. If $R_{0}>1$, then the infected equilibrium $E^{*}$ is globally asymptotically stable.

Proof. Define a Lyapunov functional

$$
L_{2}(t)=W_{2}\left(x_{1 t}, x_{2 t}, y_{1 t}, y_{2 t}, v_{t}\right)+W^{+},
$$

where

$$
\begin{aligned}
W_{2}= & e^{-\mu_{1} \tau_{1}}\left[x_{1}(t)-x_{1}^{*}-\int_{x_{1}^{*}}^{x_{1}(t)} \frac{\varphi_{1}\left(x_{1}^{*}, v^{*}\right)}{\varphi_{1}\left(\sigma, v^{*}\right)} d \sigma\right]+y_{1}(t)-y_{1}^{*} \\
& -\int_{y_{1}^{*}}^{y_{1}(t)} \frac{G_{1}\left(y_{1}^{*}\right)}{G_{1}(\sigma)} d \sigma+\alpha e^{-\mu_{2} \tau_{2}}\left[x_{2}(t)-x_{2}^{*}-\int_{x_{2}^{*}}^{x_{2}(t)} \frac{\varphi_{2}\left(x_{2}^{*}, v^{*}\right)}{\varphi_{2}\left(\sigma, v^{*}\right)} d \sigma\right] \\
& +\alpha\left(y_{2}(t)-y_{2}^{*}-\int_{y_{2}^{*}}^{y_{2}(t)} \frac{G_{2}\left(y_{2}^{*}\right)}{G_{2}(\sigma)} d \sigma\right)+\frac{a_{1}}{p_{1}}\left(v(t)-v^{*}-v^{*} \ln \frac{v(t)}{v^{*}}\right)
\end{aligned}
$$


and

$$
\begin{aligned}
W^{+}= & \varphi_{1}\left(x_{1}^{*}, v^{*}\right) e^{-\mu_{1} \tau_{1}} \int_{0}^{\tau_{1}}\left(\frac{\varphi_{1}\left(x_{1}(t-\theta), v(t-\theta)\right)}{\varphi_{1}\left(x_{1}^{*}, v^{*}\right)}-1\right. \\
& \left.-\ln \frac{\varphi_{1}\left(x_{1}(t-\theta), v(t-\theta)\right)}{\varphi_{1}\left(x_{1}^{*}, v^{*}\right)}\right) d \theta \\
& +\alpha \varphi_{2}\left(x_{2}^{*}, v^{*}\right) e^{-\mu_{2} \tau_{2}} \int_{0}^{\tau_{2}}\left(\frac{\varphi_{2}\left(x_{2}(t-\theta), v(t-\theta)\right)}{\varphi_{2}\left(x_{2}^{*}, v^{*}\right)}-1\right. \\
& \left.-\ln \frac{\varphi_{2}\left(x_{2}(t-\theta), v(t-\theta)\right)}{\varphi_{2}\left(x_{2}^{*}, v^{*}\right)}\right) d \theta .
\end{aligned}
$$

The derivative of $W_{2}$ along the solution of system (2) is

$$
\begin{aligned}
\left.\dot{W}_{2}\right|_{(2)}= & e^{-\mu_{1} \tau_{1}}\left(1-\frac{\varphi_{1}\left(x_{1}^{*}, v^{*}\right)}{\varphi_{1}\left(x_{1}, v^{*}\right)}\right)\left(\lambda_{1}-d_{1} x_{1}-\varphi_{1}\left(x_{1}, v\right)\right) \\
& +\left(1-\frac{G_{1}\left(y_{1}^{*}\right)}{G_{1}\left(y_{1}\right)}\right)\left(e^{-\mu_{1} \tau_{1}} \varphi_{1}\left(x_{1}\left(t-\tau_{1}\right), v\left(t-\tau_{1}\right)\right)-a_{1} G_{1}\left(y_{1}\right)\right) \\
& +\alpha e^{-\mu_{2} \tau_{2}}\left(1-\frac{\varphi_{2}\left(x_{2}^{*}, v^{*}\right)}{\varphi_{2}\left(x_{2}, v^{*}\right)}\right)\left(\lambda_{2}-d_{2} x_{2}-\varphi_{2}\left(x_{2}, v\right)\right) \\
& +\alpha\left(1-\frac{G_{2}\left(y_{2}^{*}\right)}{G_{2}\left(y_{2}\right)}\right)\left(e^{-\mu_{2} \tau_{2}} \varphi_{2}\left(x_{2}\left(t-\tau_{2}\right), v\left(t-\tau_{2}\right)\right)-a_{2} G_{2}\left(y_{2}\right)\right) \\
& +a_{1} G_{1}\left(y_{1}(t)\right)\left(1-\frac{v^{*}}{v}\right)+\frac{a_{1} p_{2}}{p_{1}}\left(1-\frac{v^{*}}{v}\right) G_{2}\left(y_{2}(t)\right) \\
& -\frac{a_{1}}{p_{1}}\left(1-\frac{v^{*}}{v}\right) \delta v .
\end{aligned}
$$

Similarly, we have

$$
\begin{aligned}
\left.\dot{W}^{+}\right|_{(2)}= & \varphi_{1}\left(x_{1}^{*}, v^{*}\right) e^{-\mu_{1} \tau_{1}} \frac{d}{d t} \int_{0}^{\tau_{1}}\left(\frac{\varphi_{1}\left(x_{1}(t-\theta), v(t-\theta)\right)}{\varphi_{1}\left(x_{1}^{*}, v^{*}\right)}-1\right. \\
& \left.-\ln \frac{\varphi_{1}\left(x_{1}(t-\theta), v(t-\theta)\right)}{\varphi_{1}\left(x_{1}^{*}, v^{*}\right)}\right) d \theta \\
& +\alpha \varphi_{2}\left(x_{2}^{*}, v^{*}\right) e^{-\mu_{2} \tau_{2}} \frac{d}{d t} \int_{0}^{\tau_{2}}\left(\frac{\varphi_{2}\left(x_{2}(t-\theta), v(t-\theta)\right)}{\varphi_{2}\left(x_{2}^{*}, v^{*}\right)}-1\right. \\
& \left.-\ln \frac{\varphi_{2}\left(x_{2}(t-\theta), v(t-\theta)\right)}{\varphi_{2}\left(x_{2}^{*}, v^{*}\right)}\right) d \theta \\
= & -\varphi_{1}\left(x_{1}^{*}, v^{*}\right) e^{-\mu_{1} \tau_{1}} \int_{0}^{\tau_{1}} \frac{d}{d \theta}\left(\frac{\varphi_{1}\left(x_{1}(t-\theta), v(t-\theta)\right)}{\varphi_{1}\left(x_{1}^{*}, v^{*}\right)}-1\right. \\
& \left.-\ln \frac{\varphi_{1}\left(x_{1}(t-\theta), v(t-\theta)\right)}{\varphi_{1}\left(x_{1}^{*}, v^{*}\right)}\right) d \theta \\
& -\alpha \varphi_{2}\left(x_{2}^{*}, v^{*}\right) e^{-\mu_{2} \tau_{2}} \int_{0}^{\tau_{2}} \frac{d}{d \theta}\left(\frac{\varphi_{2}\left(x_{2}(t-\theta), v(t-\theta)\right)}{\varphi_{2}\left(x_{2}^{*}, v^{*}\right)}-1\right. \\
& \left.-\ln \frac{\varphi_{2}\left(x_{2}(t-\theta), v(t-\theta)\right)}{\varphi_{2}\left(x_{2}^{*}, v^{*}\right)}\right) d \theta \\
= & e^{-\mu_{1} \tau_{1}}\left(\varphi_{1}\left(x_{1}, v\right)-\varphi_{1}\left(x_{1}\left(t-\tau_{1}\right), v\left(t-\tau_{1}\right)\right)\right)
\end{aligned}
$$




$$
\begin{aligned}
& +e^{-\mu_{1} \tau_{1}} \varphi_{1}\left(x_{1}^{*}, v^{*}\right) \ln \frac{\varphi_{1}\left(x_{1}\left(t-\tau_{1}\right), v\left(t-\tau_{1}\right)\right)}{\varphi_{1}\left(x_{1}, v\right)} \\
& +\alpha e^{-\mu_{2} \tau_{2}}\left(\varphi_{2}\left(x_{2}, v\right)-\varphi_{2}\left(x_{2}\left(t-\tau_{2}\right), v\left(t-\tau_{2}\right)\right)\right) \\
& +\alpha e^{-\mu_{2} \tau_{2}} \varphi_{2}\left(x_{2}^{*}, v^{*}\right) \ln \frac{\varphi_{2}\left(x_{2}\left(t-\tau_{2}\right), v\left(t-\tau_{2}\right)\right)}{\varphi_{2}\left(x_{2}, v\right)} .
\end{aligned}
$$

Note that

$$
\begin{aligned}
& \lambda_{i}=d_{i} x_{i}^{*}+\varphi_{i}\left(x_{i}^{*}, v^{*}\right), \quad \varphi_{i}\left(x_{i}^{*}, v^{*}\right)=e^{\mu_{i} \tau_{i}} a_{i} G_{i}\left(y_{i}^{*}\right), \quad i=1,2, \\
& \frac{\delta a_{1}}{p_{1}} v^{*}=a_{1} G_{1}\left(y_{1}^{*}\right)+\alpha a_{2} G_{2}\left(y_{2}^{*}\right), \\
& \frac{\delta a_{1}}{p_{1}} v=\left(a_{1} G_{1}\left(y_{1}^{*}\right)+\alpha a_{2} G_{2}\left(y_{2}^{*}\right)\right) \frac{v}{v^{*}}, \\
& \alpha=\frac{a_{1} p_{2}}{a_{2} p_{1}}
\end{aligned}
$$

we obtain

$$
\begin{aligned}
\left.\dot{L}_{2}\right|_{(2)}= & \dot{W}_{2}+\dot{W}^{+} \\
= & d_{1} x_{1}^{*} e^{-\mu_{1} \tau_{1}}\left(1-\frac{\varphi_{1}\left(x_{1}^{*}, v^{*}\right)}{\varphi_{1}\left(x_{1}, v^{*}\right)}\right)\left(1-\frac{x_{1}}{x_{1}^{*}}\right)+e^{-\mu_{1} \tau_{1}} \varphi_{1}\left(x_{1}^{*}, v^{*}\right)\left(1-\frac{\varphi_{1}\left(x_{1}^{*}, v^{*}\right)}{\varphi_{1}\left(x_{1}, v^{*}\right)}\right) \\
& +\alpha d_{2} x_{2}^{*} e^{-\mu_{2} \tau_{2}}\left(1-\frac{\varphi_{2}\left(x_{2}^{*}, v^{*}\right)}{\varphi_{2}\left(x_{2}, v^{*}\right)}\right)\left(1-\frac{x_{2}}{x_{2}^{*}}\right) \\
& +\alpha e^{-\mu_{2} \tau_{2}} \varphi_{2}\left(x_{2}^{*}, v^{*}\right)\left(1-\frac{\varphi_{2}\left(x_{2}^{*}, v^{*}\right)}{\varphi_{2}\left(x_{2}, v^{*}\right)}\right) \\
& +e^{-\mu_{1} \tau_{1}} \varphi_{1}\left(x_{1}^{*}, v^{*}\right) \frac{\varphi_{1}\left(x_{1}, v\right)}{\varphi_{1}\left(x_{1}, v^{*}\right)}+\alpha e^{-\mu_{2} \tau_{2}} \varphi_{2}\left(x_{2}^{*}, v^{*}\right) \frac{\varphi_{2}\left(x_{2}, v\right)}{\varphi_{2}\left(x_{2}, v^{*}\right)} \\
& -e^{-\mu_{1} \tau_{1}} \varphi_{1}\left(x_{1}^{*}, v^{*}\right) \frac{G_{1}\left(y_{1}^{*}\right) \varphi_{1}\left(x_{1}\left(t-\tau_{1}\right), v\left(t-\tau_{1}\right)\right)}{G_{1}\left(y_{1}\right) \varphi_{1}\left(x_{1}^{*}, v^{*}\right)}+a_{1} G_{1}\left(y_{1}^{*}\right) \\
& -\alpha e^{-\mu_{2} \tau_{2}} \varphi_{2}\left(x_{2}^{*}, v^{*}\right) \frac{G_{2}\left(y_{2}^{*}\right) \varphi_{2}\left(x_{2}\left(t-\tau_{2}\right), v\left(t-\tau_{2}\right)\right)}{G_{2}\left(y_{2}\right) \varphi_{2}\left(x_{2}^{*}, v^{*}\right)}+\alpha a_{2} G_{2}\left(y_{2}^{*}\right) \\
& -a_{1} G_{1}\left(y_{1}(t) \frac{v^{*}}{v}-\frac{\delta a_{1}}{p_{1}} v-\alpha a_{2} G_{2}\left(y_{2}(t)\right) \frac{v^{*}}{v}+\frac{\delta a_{1}}{p_{1}} v^{*}\right. \\
& +e^{-\mu_{1} \tau_{1}} \varphi_{1}\left(x_{1}^{*}, v^{*}\right) \ln \frac{\varphi_{1}\left(x_{1}\left(t-\tau_{1}\right), v\left(t-\tau_{1}\right)\right)}{\varphi_{1}\left(x_{1}, v\right)} \\
& +\alpha e^{-\mu_{2} \tau_{2}} \varphi_{2}\left(x_{2}^{*}, v^{*}\right) \ln \frac{\varphi_{2}\left(x_{2}\left(t-\tau_{2}, v\left(t-\tau_{2}\right)\right)\right.}{\varphi_{2}\left(x_{2}, v\right)}
\end{aligned}
$$


which is equal to

$$
\begin{aligned}
& e^{-\mu_{1} \tau_{1}} d_{1} x_{1}^{*}\left(1-\frac{\varphi_{1}\left(x_{1}^{*}, v^{*}\right)}{\varphi_{1}\left(x_{1}, v^{*}\right)}\right)\left(1-\frac{x_{1}}{x_{1}^{*}}\right) \\
& +\alpha e^{-\mu_{2} \tau_{2}} d_{2} x_{2}^{*}\left(1-\frac{\varphi_{2}\left(x_{2}^{*}, v^{*}\right)}{\varphi_{2}\left(x_{2}, v^{*}\right)}\right)\left(1-\frac{x_{2}}{x_{2}^{*}}\right) \\
& +a_{1} G_{1}\left(y_{1}^{*}\right)\left(1-\frac{G_{1}\left(y_{1}^{*}\right) \varphi_{1}\left(x_{1}\left(t-\tau_{1}\right), v\left(t-\tau_{1}\right)\right)}{G_{1}\left(y_{1}\right) \varphi_{1}\left(x_{1}^{*}, v^{*}\right)}\right. \\
& \left.+\ln \frac{G_{1}\left(y_{1}^{*}\right) \varphi_{1}\left(x_{1}\left(t-\tau_{1}\right), v\left(t-\tau_{1}\right)\right)}{G_{1}\left(y_{1}\right) \varphi_{1}\left(x_{1}^{*}, v^{*}\right)}\right) \\
& +a_{1} G_{1}\left(y_{1}^{*}\right)\left(1-\frac{v^{*} G_{1}\left(y_{1}(t)\right)}{v G_{1}\left(y_{1}^{*}\right)}+\ln \frac{v^{*} G_{1}\left(y_{1}(t)\right)}{v G_{1}\left(y_{1}^{*}\right)}\right) \\
& +a_{1} G_{1}\left(y_{1}^{*}\right)\left(1-\frac{\varphi_{1}\left(x_{1}^{*}, v^{*}\right)}{\varphi_{1}\left(x_{1}, v^{*}\right)}+\ln \frac{\varphi_{1}\left(x_{1}^{*}, v^{*}\right)}{\varphi_{1}\left(x_{1}, v^{*}\right)}\right) \\
& +a_{1} G_{1}\left(y_{1}^{*}\right)\left(1-\frac{v \varphi_{1}\left(x_{1}, v^{*}\right)}{v^{*} \varphi_{1}\left(x_{1}, v\right)}+\ln \frac{v \varphi_{1}\left(x_{1}, v^{*}\right)}{v^{*} \varphi_{1}\left(x_{1}, v\right)}\right) \\
& +a_{1} G_{1}\left(y_{1}^{*}\right)\left(-1-\frac{v}{v^{*}}+\frac{v \varphi_{1}\left(x_{1}, v^{*}\right)}{v^{*} \varphi_{1}\left(x_{1}, v\right)}+\frac{\varphi_{1}\left(x_{1}, v\right)}{\varphi_{1}\left(x_{1}, v^{*}\right)}\right) \\
& +\alpha a_{2} G_{2}\left(y_{2}^{*}\right)\left(1-\frac{G_{2}\left(y_{2}^{*}\right) \varphi_{2}\left(x_{2}\left(t-\tau_{2}\right), v\left(t-\tau_{2}\right)\right)}{G_{2}\left(y_{2}\right) \varphi_{2}\left(x_{2}^{*}, v^{*}\right)}\right. \\
& \left.+\ln \frac{G_{2}\left(y_{2}^{*}\right) \varphi_{2}\left(x_{2}\left(t-\tau_{2}\right), v\left(t-\tau_{2}\right)\right)}{G_{2}\left(y_{2}\right) \varphi_{2}\left(x_{2}^{*}, v^{*}\right)}\right) \\
& +\alpha a_{2} G_{2}\left(y_{2}^{*}\right)\left(1-\frac{v^{*} G_{2}\left(y_{2}(t)\right)}{v G_{2}\left(y_{2}^{*}\right)}+\ln \frac{v^{*} G_{2}\left(y_{2}(t)\right)}{v G_{2}\left(y_{2}^{*}\right)}\right) \\
& +\alpha a_{2} G_{2}\left(y_{2}^{*}\right)\left(1-\frac{\varphi_{2}\left(x_{2}^{*}, v^{*}\right)}{\varphi_{2}\left(x_{2}, v^{*}\right)}+\ln \frac{\varphi_{2}\left(x_{2}^{*}, v^{*}\right)}{\varphi_{2}\left(x_{2}, v^{*}\right)}\right) \\
& +\alpha a_{2} G_{2}\left(y_{2}^{*}\right)\left(1-\frac{v \varphi_{2}\left(x_{2}, v^{*}\right)}{v^{*} \varphi_{2}\left(x_{2}, v\right)}+\ln \frac{v \varphi_{2}\left(x_{2}, v^{*}\right)}{v^{*} \varphi_{2}\left(x_{2}, v\right)}\right) \\
& +\alpha a_{2} G_{2}\left(y_{2}^{*}\right)\left(-1-\frac{v}{v^{*}}+\frac{v \varphi_{2}\left(x_{2}, v^{*}\right)}{v^{*} \varphi_{2}\left(x_{2}, v\right)}+\frac{\varphi_{2}\left(x_{2}, v\right)}{\varphi_{2}\left(x_{2}, v^{*}\right)}\right) .
\end{aligned}
$$

By the concavity and monotonicity of the function $\varphi_{i}\left(x_{i}, v\right)$ with respect to $v$, we have

$$
\begin{aligned}
& -1-\frac{v}{v^{*}}+\frac{v \varphi_{i}\left(x_{i}, v^{*}\right)}{v^{*} \varphi_{i}\left(x_{i}, v\right)}+\frac{\varphi_{i}\left(x_{i}, v\right)}{\varphi_{i}\left(x_{i}, v^{*}\right)} \\
& =\left(\frac{\varphi_{i}\left(x_{i}, v^{*}\right)}{\varphi_{i}\left(x_{i}, v\right)}-1\right)\left(\frac{v}{v^{*}}-\frac{\varphi_{i}\left(x_{i}, v\right)}{\varphi_{i}\left(x_{i}, v^{*}\right)}\right) \\
& \leq 0 . \quad i=1,2 .
\end{aligned}
$$

From the monotonicity of $\varphi_{i}\left(x_{i}, v\right)$ with respect to $x_{i}$, we have

$$
\left(1-\frac{x_{i}}{x_{i}^{*}}\right)\left(1-\frac{\varphi_{i}\left(x_{i}^{*}, v^{*}\right)}{\varphi_{i}\left(x_{i}, v^{*}\right)}\right) \leq 0, \quad i=1,2
$$

Let

$$
g(z)=z-1-\ln z, \quad z \in R^{+}
$$

It is nonnegative for any $z>0$, and $g(z)=0$ if and only if $z=1$. Therefore, we 
obtain

$$
\begin{aligned}
\left.\dot{L}_{2}\right|_{(2)}= & e^{-\mu_{1} \tau_{1}} d_{1} x_{1}^{*}\left(1-\frac{\varphi_{1}\left(x_{1}^{*}, v^{*}\right)}{\varphi_{1}\left(x_{1}, v^{*}\right)}\right)\left(1-\frac{x_{1}}{x_{1}^{*}}\right) \\
& +\alpha e^{-\mu_{2} \tau_{2}} d_{2} x_{2}^{*}\left(1-\frac{\varphi_{2}\left(x_{2}^{*}, v^{*}\right)}{\varphi_{2}\left(x_{2}, v^{*}\right)}\right)\left(1-\frac{x_{2}}{x_{2}^{*}}\right) \\
& -a_{1} G_{1}\left(y_{1}^{*}\right) g\left(\frac{G_{1}\left(y_{1}^{*}\right) \varphi_{1}\left(x_{1}\left(t-\tau_{1}\right), v\left(t-\tau_{1}\right)\right)}{G_{1}\left(y_{1}\right) \varphi_{1}\left(x_{1}^{*}, v^{*}\right)}\right) \\
& -a_{1} G_{1}\left(y_{1}^{*}\right)\left(g\left(\frac{v^{*} G_{1}\left(y_{1}\right)}{v G_{1}\left(y_{1}^{*}\right)}\right)+g\left(\frac{\varphi_{1}\left(x_{1}^{*}, v^{*}\right)}{\varphi_{1}\left(x_{1}, v^{*}\right)}\right)+g\left(\frac{v \varphi_{1}\left(x_{1}, v^{*}\right)}{v^{*} \varphi_{1}\left(x_{1}, v\right)}\right)\right) \\
& +a_{1} G_{1}\left(y_{1}^{*}\right)\left(\frac{\varphi_{1}\left(x_{1}, v^{*}\right)}{\varphi_{1}\left(x_{1}, v\right)}-1\right)\left(\frac{v}{v^{*}}-\frac{\varphi_{1}\left(x_{1}, v\right)}{\varphi_{1}\left(x_{1}, v^{*}\right)}\right) \\
& -\alpha a_{2} G_{2}\left(y_{2}^{*}\right) g\left(\frac{G_{2}\left(y_{2}^{*}\right) \varphi_{2}\left(x_{2}\left(t-\tau_{2}\right), v\left(t-\tau_{2}\right)\right)}{G_{2}\left(y_{2}\right) \varphi_{2}\left(x_{2}^{*}, v^{*}\right)}\right) \\
& -\alpha a_{2} G_{2}\left(y_{2}^{*}\right)\left(g\left(\frac{v^{*} G_{2}\left(y_{2}\right)}{v G_{2}\left(y_{2}^{*}\right)}\right)+g\left(\frac{\varphi_{2}\left(x_{2}^{*}, v^{*}\right)}{\varphi_{2}\left(x_{2}, v^{*}\right)}\right)+g\left(\frac{v \varphi_{2}\left(x_{2}, v^{*}\right)}{v^{*} \varphi_{2}\left(x_{2}, v\right)}\right)\right) \\
& +\alpha a_{2} G_{2}\left(y_{2}^{*}\right)\left(\frac{\varphi_{2}\left(x_{2}, v^{*}\right)}{\varphi_{2}\left(x_{2}, v\right)}-1\right)\left(\frac{v}{v^{*}}-\frac{\varphi_{2}\left(x_{2}, v\right)}{\varphi_{2}\left(x_{2}, v^{*}\right)}\right) .
\end{aligned}
$$

Thus, $\left.\dot{L}_{2}\right|_{(2)} \leq 0$ for all $\left(x_{1}, x_{2}, y_{1}, y_{2}, v\right)$. The largest invariant subset of $\left\{\left.\dot{L}_{2}\right|_{(2)}=0\right\}$ is $\left\{E^{*}\right\}$. By LaSalle Invariance Principle and a similar argument in the proof of Theorem 3.1 , we show that $E^{*}$ is globally asymptotically stable.

\section{A general model with $n$ target cell populations}

In this section, we extend the model with two target cell populations to include $n$ populations. The model becomes a $(2 n+1)$-dimensional system with $n$ time delays.

$$
\left\{\begin{array}{l}
\dot{x}_{i}(t)=\lambda_{i}-d_{i} x_{i}(t)-\varphi_{i}\left(x_{i}(t), v(t)\right), \\
\dot{y}_{i}(t)=e^{-\mu_{i} \tau_{i}} \varphi_{i}\left(x_{i}\left(t-\tau_{i}\right), v\left(t-\tau_{i}\right)\right)-a_{i} G_{i}\left(y_{i}\right), \quad i=1,2, \cdots, n . \\
\dot{v}(t)=\sum_{i=1}^{n} p_{i} G_{i}\left(y_{i}(t)\right)-\delta v(t) .
\end{array}\right.
$$

This system describes the interaction of virus with $n$ classes of target cells. The state variables $x_{i}, y_{i}, v$ and all the parameters of system (17) have the same biological meaning as those in Section 2.

The initial condition for system (17) is given by

$$
x_{i}(\theta)=\varphi_{i}(\theta), y_{i}(\theta)=\xi_{i}(\theta), v(\theta)=\gamma(\theta), \quad i=1,2, \cdots, n .
$$

Here $\varphi=\left(\varphi_{1}, \cdots, \varphi_{n}, \xi_{1}, \cdots, \xi_{n}, \gamma\right)^{T} \in C\left([-\tau, 0], R_{+}^{2 n+1}\right)$, the Banach space of continuous functions from $[-\tau, 0]$ to $R_{+}^{2 n+1}$, where $\tau=\max \left\{\tau_{1}, \cdots, \tau_{n}\right\}$. 
Similarly, the solutions of system (17) are all nonnegative and bounded. System (17) has an infection-free equilibrium

$$
P^{0}=\left(x_{1}^{0}, \cdots, x_{n}^{0}, y_{1}^{0}, \cdots, y_{n}^{0}, 0\right), \text { where } x_{i}^{0}=\frac{\lambda_{i}}{d_{i}}, y_{i}^{0}=0, i=1,2, \cdots, n .
$$

When $\widetilde{R}_{0}>1$, it has an infected equilibrium $P^{*}=\left(\widetilde{x}_{1}, \cdots, \widetilde{x}_{n}, \widetilde{y}_{1}, \cdots, \widetilde{y}_{n}, \widetilde{v}\right)$, where

$$
\widetilde{R}_{0}=\sum_{i=1}^{n} \frac{p_{i}}{\delta a_{i}} \cdot \frac{\partial \varphi_{i}\left(x_{i}^{0}, 0\right)}{\partial v} e^{-\mu_{i} \tau_{i}} .
$$

For the general model, we have the following global stability results for the steady states $P^{0}$ and $P^{*}$ by constructing Lyapunov functionals.

Theorem 4.1 Suppose that the conditions $\left(\mathrm{H}_{1}\right)-\left(\mathrm{H}_{5}\right)$ hold.

(i) If $\widetilde{R}_{0} \leq 1$, then the infection-free equilibrium $P^{0}$ of system (17) is globally asymptotically stable.

(ii) If $\widetilde{R}_{0}>1$, then the infected equilibrium $P^{*}$ of system (17) is globally asymptotically stable.

Proof. (i) Let

$$
\widetilde{L}_{1}(t)=U_{1}\left(x_{1 t}, \cdots, x_{n t}, y_{1 t}, \cdots, y_{n t}, v_{t}\right)+U_{+},
$$

where

$$
\begin{aligned}
U_{1}= & \sum_{i=1}^{n} \alpha_{i} e^{-\mu_{i} \tau_{i}}\left(x_{i}(t)-x_{i}^{0}-\int_{x_{i}^{0}}^{x_{i}(t)} \lim _{v \rightarrow 0^{+}} \frac{\varphi_{i}\left(x_{i}^{0}, v\right)}{\varphi_{i}(\sigma, v)} d \sigma\right) \\
& +\sum_{i=1}^{n} \alpha_{i} y_{i}(t)+\frac{a_{1}}{p_{1}} e^{n_{1} \omega_{1}} v(t), \\
U_{+} & =\sum_{i=1}^{n} \alpha_{i} e^{-\mu_{i} \tau_{i}} \int_{0}^{\tau_{i}} \varphi_{i}\left(x_{i}(t-\theta), v(t-\theta)\right) d \theta,
\end{aligned}
$$

and $\alpha_{i}=\frac{a_{1} p_{i}}{a_{i} p_{1}}$

Using $\lambda_{i}=d_{i} x_{i}^{0}$ and the concavity of $\varphi_{i}\left(x_{i}, v\right)$ with respect to $v$, we have

$$
\begin{aligned}
& \frac{p_{i} e^{-\mu_{i} \tau_{i}}}{\delta a_{i}} \cdot \frac{\varphi_{i}\left(x_{i}, v\right)}{v} \cdot \lim _{v \rightarrow 0^{+}} \frac{\varphi_{i}\left(x_{i}^{0}, v\right)}{\varphi_{i}\left(x_{i}, v\right)} \\
& =\frac{p_{i}}{\delta a_{i}} \cdot \frac{\varphi_{i}\left(x_{i}, v\right)}{v} \cdot \frac{\frac{\partial \varphi_{i}\left(x_{i}^{0}, 0\right)}{\partial v}}{\frac{\partial \varphi_{i}\left(x_{i}, 0\right)}{\partial v}} \cdot e^{-\mu_{i} \tau_{i}} \\
& \leq \frac{p_{i}}{\delta a_{i}} \cdot \frac{\partial \varphi_{i}\left(x_{i}^{0}, 0\right)}{\partial v} \cdot e^{-\mu_{i} \tau_{i}} .
\end{aligned}
$$


From $\left(H_{4}\right)$, we get

$$
\begin{aligned}
& \left(\frac{x_{i}^{0}}{x_{i}}-1\right)\left(1-\lim _{v \rightarrow 0^{+}} \frac{\varphi_{i}\left(x_{i}^{0}, v\right)}{\varphi_{i}\left(x_{i}, v\right)}\right) \\
& =\left(\frac{x_{i}^{0}}{x_{i}}-1\right)\left(1-\frac{\frac{\partial \varphi_{i}\left(x_{i}^{0}, 0\right)}{\partial v}}{\frac{\partial \varphi_{i}\left(x_{i}, 0\right)}{\partial v}}\right) \\
& \leq 0 .
\end{aligned}
$$

Thus, it follows from (20) and (21) that

$$
\begin{aligned}
\left.\dot{\widetilde{L}}_{1}(t)\right|_{(17)}= & \dot{U}_{1}+\dot{U}_{+} \\
= & \sum_{i=1}^{n} \alpha_{i} e^{-\mu_{i} \tau_{i}} d_{i} x_{i}\left(\frac{x_{i}^{0}}{x_{i}}-1\right)\left(1-\lim _{v \rightarrow 0^{+}} \frac{\varphi_{i}\left(x_{i}^{0}, v\right)}{\varphi_{i}\left(x_{i}, v\right)}\right) \\
& +\sum_{i=1}^{n} \alpha_{i} e^{-\mu_{i} \tau_{i}} \varphi_{i}\left(x_{i}, v\right) \lim _{v \rightarrow 0^{+}} \frac{\varphi_{i}\left(x_{i}^{0}, v\right)}{\varphi_{i}\left(x_{i}, v\right)}-\frac{\delta a_{1}}{p_{1}} v(t) \\
= & \sum_{i=1}^{n} \alpha_{i} e^{-\mu_{i} \tau_{i}} d_{i} x_{i}\left(\frac{x_{i}^{0}}{x_{i}}-1\right)\left(1-\lim _{v \rightarrow 0^{+}} \frac{\varphi_{i}\left(x_{i}^{0}, v\right)}{\varphi_{i}\left(x_{i}, v\right)}\right) \\
& +\frac{\delta a_{1}}{p_{1}} v(t)\left[\sum_{i=1}^{n} \frac{\alpha_{i} p_{1} e^{-\mu_{i} \tau_{i}}}{\delta a_{1}} \cdot \frac{\varphi_{i}\left(x_{i}, v\right)}{v} \cdot \lim _{v \rightarrow 0^{+}} \frac{\varphi_{i}\left(x_{i}^{0}, v\right)}{\varphi_{i}\left(x_{i}, v\right)}-1\right] \\
= & \sum_{i=1}^{n} \alpha_{i} e^{-\mu_{i} \tau_{i}} d_{i} x_{i}\left(\frac{x_{i}^{0}}{x_{i}}-1\right)\left(1-\lim _{v \rightarrow 0^{+}} \frac{\varphi_{i}\left(x_{i}^{0}, v\right)}{\varphi_{i}\left(x_{i}, v\right)}\right) \\
& +\frac{\delta a_{1}}{p_{1}} v(t)\left[\sum_{i=1}^{n} \frac{p_{i}}{\delta a_{i}} \cdot \frac{\varphi_{i}\left(x_{i}, v\right)}{v} \cdot \frac{\frac{\partial \varphi_{i}\left(x_{i}^{0}, 0\right)}{\partial v}}{\frac{\partial \varphi_{i}\left(x_{i}, 0\right)}{\partial v}} \cdot e^{-\mu_{i} \tau_{i}}-1\right] \\
\leq & \sum_{i=1}^{n} \alpha_{i} e^{-\mu_{i} \tau_{i}} d_{i} x_{i}\left(\frac{x_{i}^{0}}{x_{i}}-1\right)\left(1-\lim _{v \rightarrow 0^{+}} \frac{\varphi_{i}\left(x_{i}^{0}, v\right)}{\varphi_{i}\left(x_{i}, v\right)}\right) \\
& +\frac{\delta a_{1}}{p_{1}} v(t)\left(\widetilde{R}_{0}-1\right) .
\end{aligned}
$$

By LaSalle Invariance Principle and a similar argument in the proof Theorem 3.1, we show that the infection-free equilibrium $P^{0}$ is globally asymptotically stable.

(ii) Define

$$
\widetilde{L}_{2}(t)=U_{2}\left(x_{1 t}, \cdots, x_{n t}, y_{1 t}, \cdots, y_{n t}, v_{t}\right)+U^{+}
$$

where

$$
\begin{aligned}
& U_{2}= \sum_{i=1}^{n} \alpha_{i} e^{-\mu_{i} \tau_{i}}\left(x_{i}(t)-\widetilde{x}_{i}-\int_{\widetilde{x}_{i}}^{x_{i}(t)} \lim _{v \rightarrow 0^{+}} \frac{\varphi_{i}\left(\widetilde{x}_{i}, \widetilde{v}\right)}{\varphi_{i}(\sigma, \widetilde{v})} d \sigma\right) \\
&+\sum_{i=1}^{n} \alpha_{i}\left(y_{i}(t)-\widetilde{y}_{i}-\int_{\widetilde{y}_{i}}^{y_{i}(t)} \frac{G_{i}\left(\widetilde{y}_{i}\right)}{G_{i}(\sigma)} d \sigma\right) \\
&+\frac{a_{1}}{p_{1}}\left(v(t)-\widetilde{v}-\widetilde{v} \ln \frac{v(t)}{\widetilde{v}}\right), \\
& U^{+}=\sum_{i=1}^{n} \alpha_{i} \varphi_{i}\left(\widetilde{x}_{i}, \widetilde{v}\right) e^{-\mu_{i} \tau_{i}} \int_{0}^{\tau_{i}}\left(\frac{\varphi_{i}\left(x_{i}(t-\theta), v(t-\theta)\right)}{\varphi_{i}\left(x_{i}, v\right)}-1-\ln \frac{\varphi_{i}\left(x_{i}(t-\theta), v(t-\theta)\right)}{\varphi_{i}\left(x_{i}, \widetilde{v}\right)}\right) d \theta .
\end{aligned}
$$


The derivative of $\widetilde{L}_{2}$ along the solution of system (17) is

$$
\begin{aligned}
\left.\dot{\widetilde{L}}_{2}(t)\right|_{(17)}= & \dot{U}_{2}+\dot{U}^{+} \\
= & \sum_{i=1}^{n} \alpha_{i} e^{-\mu_{i} \tau_{i}} d_{i} \widetilde{x}_{i}\left(1-\frac{\varphi_{i}\left(\widetilde{x}_{i}, \widetilde{v}\right)}{\varphi_{i}\left(x_{i}, \widetilde{v}\right)}\right)\left(1-\frac{x_{i}}{\widetilde{x}_{i}}\right) \\
& -\sum_{i=1}^{n} \alpha_{i} a_{i} G_{i}\left(\widetilde{y}_{i}\right) g\left(\frac{G_{i}\left(\widetilde{y}_{i}\right) \varphi_{i}\left(x_{i}\left(t-\tau_{i}\right), v\left(t-\tau_{i}\right)\right)}{G_{i}\left(y_{i}\right) \varphi_{i}\left(\widetilde{x}_{i}, \widetilde{v}\right)}\right) \\
& -\sum_{i=1}^{n} \alpha_{i} a_{i} G_{i}\left(\widetilde{y}_{i}\right) g\left(\frac{\widetilde{v} g_{i}\left(y_{i}\right)}{v G_{i}\left(\widetilde{y}_{i}\right)}\right) \\
& -\sum_{i=1}^{n} \alpha_{i} a_{i} G_{i}\left(\widetilde{y}_{i}\right)\left(g\left(\frac{\varphi_{i}\left(\widetilde{x}_{i}, \widetilde{v}\right)}{\varphi_{i}\left(x_{i}, \widetilde{v}\right)}\right)+g\left(\frac{v \varphi_{i}\left(x_{i}, \widetilde{v}\right)}{\widetilde{v} \varphi_{i}\left(x_{i}, v\right)}\right)\right) \\
& +\sum_{i=1}^{n} \alpha_{i} a_{i} G_{i}\left(\widetilde{y}_{i}\right) g\left(\frac{\varphi_{i}\left(x_{i}, \widetilde{v}\right)}{\varphi_{i}\left(x_{i}, v\right)}-1\right)\left(\frac{v}{\widetilde{v}}-\frac{\varphi_{i}\left(x_{i}, v\right)}{\varphi_{i}\left(x_{i}, \widetilde{v}\right)}\right) .
\end{aligned}
$$

The following three conditions are satisfied.

(a)

$$
\begin{aligned}
& -1-\frac{v}{\widetilde{v}}+\frac{v \varphi_{i}\left(x_{i}, \widetilde{v}\right)}{\widetilde{v} \varphi_{i}\left(x_{i}, v\right)}+\frac{\varphi_{i}\left(x_{i}, v\right)}{\varphi_{i}\left(x_{i}, \widetilde{v}\right)} \\
& =\left(\frac{\varphi_{i}\left(x_{i}, \widetilde{v}\right)}{\varphi_{i}\left(x_{i}, v\right)}-1\right)\left(\frac{v}{\widetilde{v}}-\frac{\varphi_{i}\left(x_{i}, v\right)}{\varphi_{i}\left(x_{i}, \widetilde{v}\right)}\right) \\
& \leq 0, \quad i=1,2, \cdots, n .
\end{aligned}
$$

(b) By the monotonicity of $\varphi_{i}\left(x_{i}, v\right)$ with respect to $x_{i}$, we have

$$
\left(1-\frac{x_{i}}{\widetilde{x}_{i}}\right)\left(1-\frac{\varphi_{i}\left(\widetilde{x}_{i}, \widetilde{v}\right)}{\varphi_{i}\left(x_{i}, \widetilde{v}\right)}\right) \leq 0, \quad i=1,2, \cdots, n .
$$

(c) $g(z)=z-1-\ln z \geq 0, z \in R^{+}$and $g(z)=0$ if and only if $z=1$.

It follows from $(27)-(29)$ that $\left.\dot{\tilde{L}}_{2}(t)\right|_{(17)} \leq 0$ for all $x_{1}, \cdots, x_{n}, y_{1}, \cdots, y_{n}, v>0$. Again by LaSalle Invariance Principle, we know that $P^{*}$ is globally asymptotically stable.

\section{$5 \quad$ Numerical simulation}

In this section, we perform computer simulation of system (2) with specific rates of viral infection and cell death. We choose $\varphi_{i}\left(x_{i}(t), v(t)\right)=\beta_{i} x_{i}(t) v(t)$ and $G_{i}\left(y_{i}\right)=$ $y_{i}, i=1,2$, which are widely used in viral dynamic models. In Fig. 1, we showed a case in which viral infection is predicted to die out. We chose $\lambda_{1}=\lambda_{2}=1.0 \times 10^{4}$ cells $\mathrm{ml}^{-1}$ day $^{-1}, d_{1}=0.01$ day $^{-1}, d_{2}=0.01$ day $^{-1}, \beta_{1}=2.4 \times 10^{-8} \mathrm{ml} \mathrm{day}^{-1}$, 
$\beta_{2}=2.4 \times 10^{-9} \mathrm{ml}$ day $^{-1}, \delta=23$ day $^{-1}, \mu_{1}=0.05$ day $^{-1}, \mu_{2}=0.02$ day $^{-1}$, $a_{1}=0.5$ day $^{-1}, a_{2}=0.1$ day $^{-1}, \tau_{1}=0.25$ days, $\tau_{2}=0.5$ days, $p_{1}=150$ per cell per day, and $p_{2}=50$ per cell per day. Most of them were chosen from existing literature $[37,39,41]$. The viral infection rate and viral production rate include the effect of antiretroviral therapy, which can block viral infection or production. With these parameters, the basic reproductive number is $R_{0} \approx 0.361<1$. By Theorem 3.1 , the infection-free equilibrium $E^{0}$ is globally asymptotically stable. Fig. 1(a) and (b) show the dynamics of cells and viral load, respectively. When we change some parameters to $\beta_{1}=2.4 \times 10^{-7} \mathrm{ml}$ day $^{-1}, \beta_{2}=2.4 \times 10^{-8} \mathrm{ml} \mathrm{day}^{-1}, p_{2}=8$ per cell per day, and let the other parameters remain unchanged, the basic reproductive number becomes $R_{0} \approx 3.17>1$. It follows from Theorem 3.2 that the infected steady state $E^{*}$ is globally asymptotically stable. The dynamics of cells and viral load are shown in Fig. 2.

To study the influence of time delays $\left(\tau_{1}\right.$ and $\left.\tau_{2}\right)$ on the dynamics of viral load, we considered the following two cases. In Fig. 3(a), we increased $\tau_{1}$ (i.e., $\tau_{1}=0.25,1$, or 2 days) but fixed $\tau_{2}=1$ day and other parameter values as in Fig. 2 . We found that increasing the time delay does not have a visible influence on the magnitude of the viral peak or the steady state, but postpones the time to reach the viral load peak. If we increased $\tau_{2}$ (i.e., $\tau_{2}=1,5$, or 10 days) but fixed $\tau_{1}=0.25$ days, we did not observe any change in the viral load (see Fig. 3(b)). This is because of the minor contribution from infected macrophages to the total viral load. We will further investigate the relative contribution to viral production from the two cell populations below.

To evaluate the relative contribution to viral load from infected CD4 ${ }^{+} \mathrm{T}$ cells and macrophages, we plotted two ratios

$$
\frac{p_{1} y_{1}(t)}{p_{1} y_{1}(t)+p_{2} y_{2}(t)} \text { and } \frac{p_{2} y_{1}(t)}{p_{1} y_{1}(t)+p_{2} y_{2}(t)}
$$

which represent the proportion of viral production from productively infected CD4 ${ }^{+}$ $\mathrm{T}$ cells and macrophages, respectively. Parameter values were chosen to be the same as those used in Fig. 3(b). In Fig. 4(a), $\tau_{2}$ was fixed to be 1 day and $\tau_{1}$ increased from 0.25 to 2 days. In Fig. 4(b), $\tau_{1}$ was fixed to be 0.25 days and $\tau_{2}$ increased from 0.5 to 5 days. In both cases, viral production from infected $\mathrm{CD} 4^{+} \mathrm{T}$ cells accounts for the majority of viral load. When $\tau_{1}$ increases from 0.25 to 2 days, the contribution from infected $\mathrm{CD} 4^{+} \mathrm{T}$ cells becomes less. However, it is still much higher than the contribution from macrophages. Thus, the plot of relative contributions does not have a difference at the steady state level (Fig. 4(a)). When $\tau_{2}$ increases from 0.5 to 5 days, the contribution from infected macrophages becomes less. This explains why a larger value of $\tau_{2}$ leads to a lower contribution from macrophages and a higher contribution from $\mathrm{CD} 4^{+} \mathrm{T}$ cells (see Fig. 4(b)). 


\section{Conclusion and discussion}

Most of existing mathematical models of HIV infection study the viral infection and production in $\mathrm{CD}^{+}{ }^{+} \mathrm{T}$ cells. However, HIV can also infect other cells such as macrophages. In this paper, we study the global stability of the equilibrium points of an HIV model with two target cell populations. The model includes general nonlinear rates of viral infection and cell death. Time delays between viral entry into cells and production of new virions are also included in the model. We derived the basic reproductive number of the model and performed the global stability analysis of the steady states. The infection-free steady state is globally asymptotically stable when the basic reproductive number is less than or equal to 1 , and the infected steady state is globally asymptotically stable when the basic reproductive number is greater than 1. Our analysis extends some existing results in the literature. For example, the global stability was analyzed for a model with one target cell population [14]. If the general nonlinear rate of infected cell death is chosen to be $a_{i} G_{i}\left(y_{i}\right)=a_{i} y_{i}$ and the rate of viral infection is chosen to be $\varphi_{i}\left(x_{i}, v\right)=\beta_{i} x_{i} v, \beta_{i} x_{i} v /(1+v)$, or $\beta_{i} x_{i} v /\left(1+m_{i} x_{i}+b_{i} v\right)$, then the model studied here becomes those in refs. $[7,8]$.

Mathematical analysis of the model suggests that the virus is predicted to be completely cleared from infected individuals if the basic reproductive number is reduced to below 1 by antiretroviral therapy (which can reduce the infection rate and viral production rate). However, current antiretroviral therapy cannot eradicate the virus. The major obstacle to viral elimination is the existence of latently infected CD4 ${ }^{+}$ $\mathrm{T}$ cells, which cannot be affected by treatment or the immune response but can be activated to produce new virions. Mathematical models have been constructed to study the dynamics of low-level viral load persistence and latently infected cells (see the review in ref. [39]). Models have also been developed to study the emergence of drug resistance during treatment [38] and HIV immune responses [3], which are not included here. The major goal of this paper is to study the dynamics of HIV infection in multiple target cell populations.

We also extend the model with two target cell populations to a general model with $n$ populations. Similar global stability results are obtained for the general model. Numerical simulations are performed to illustrate the stability results. The simulation also shows that virus is mainly produced from infected $\mathrm{CD} 4^{+} \mathrm{T}$ cells. Thus, the time delay between viral entry into macrophages and viral production does not have an influence in the dynamics of viral load. The delay between viral entry into $\mathrm{CD}^{+}{ }^{+} \mathrm{T}$ cells does not change the magnitude of the viral peak and the steadystate viral load, but postpones the time to reach the viral peak. This is consistent with the results in ref. [33] in which a model including two time delays (one is the intracellular delay and the other is the time for the adaptive immune response to emerge) was analyzed and fit to patient data during primary HIV infection. 


\section{Acknowledgment}

This work is supported by the NNSF of China (No.11301453), Postdoctoral Science Foundation of China (2014M562366), Postdoctoral Science Foundation of Shaanxi Province (2014010), the Universities Young Teachers Program of Henan Province (2014GGJS-093), and Doctoral Scientific Research Startup Fund of Xinyang Normal University (2014033). L. Rong is supported by the NSF grant DMS-1349939. We thank a reviewer for the valuable comments that improved this manuscript.

\section{References}

[1] R. M. Andersen, R. M. May, Epidemiological parameters of HIV transmission, Nature 333 (6173) (1988) 514-519.

[2] R. M. Anderson, R. M. May, The population dynamics of microparasites and their invertebrate hosts, Philosophical Transactions of the Royal Society B: Biological Sciences 291 (1054) (1981) 451-524.

[3] D. Burg, L. Rong, A.U. Neumann, H. Dahari, Mathematical modeling of viral kinetics under immune control during primary HIV-1 infection, Journal of Theoretical Biology 259 (2009) 751-759.

[4] D. S. Burke, Human hiv vaccine trials: does antibody-dependent enhancement pose a genuine risk, Perspectives in Biology and Medicine 35 (4) (1992) 511-530.

[5] D. S. Callaway, A. S. Perelson, HIV-1 infection and low steady state viral loads, Bulletin of Mathematical Biology 64 (1) (2002) 29-64.

[6] R. V. Culshaw, S. Ruan, A delay-differential equation model of HIV infection of cd4 ${ }^{+}$T-cells, Mathematical Biosciences 165 (1) (2000) 27-39.

[7] A. Elaiw, Global properties of a class of virus infection models with multitarget cells, Nonlinear Dynamics 69 (1-2) (2012) 423-435.

[8] A. Elaiw, I. Hassanien, S. Azoz, Global stability of HIV infection models with intracellular delays, Journal of the Korean Mathematical Society 49 (4) (2012) 779-794.

[9] P. Georgescu, Y.-H. Hsieh, Global stability for a virus dynamics model with nonlinear incidence of infection and removal, SIAM Journal on Applied Mathematics 67 (2) (2006) 337-353.

[10] S. A. Gourley, Y. Kuang, J. D. Nagy, Dynamics of a delay differential equation model of hepatitis B virus infection, Journal of Biological Dynamics 2 (2) (2008) 140-153.

[11] J. K. Hale, Introduction to functional differential equations, vol. 99, Springer, 1993. 
[12] A. Herz, S. Bonhoeffer, R. M. Anderson, R. M. May, M. A. Nowak, Viral dynamics in vivo: limitations on estimates of intracellular delay and virus decay, Proceedings of the National Academy of Sciences 93 (14) (1996) 7247-7251.

[13] S. E. Holte, A. J. Melvin, J. I. Mullins, N. H. Tobin, L. M. Frenkel, Densitydependent decay in HIV-1 dynamics, Journal of Acquired Immune Deficiency Syndromes 41 (3) (2006) 266-276.

[14] G. Huang, Y. Takeuchi, W. Ma, Lyapunov functionals for delay differential equations model of viral infections, SIAM Journal on Applied Mathematics 70 (7) (2010) 2693-2708.

[15] T. Kajiwara, T. Sasaki, Y. Takeuchi, Construction of lyapunov functionals for delay differential equations in virology and epidemiology, Nonlinear Analysis: Real World Applications 13 (4) (2012) 1802-1826.

[16] S. Koenig, H.E. Gendelman, J.M. Orenstein, M.C. Dal Canto, G.H. Pezeshkpour, M. Yungbluth, F. Janotta, A. Aksamit, M.A. Martin, A.S. Fauci, Detection of AIDS virus in macrophages in brain tissue from AIDS patients with encephalopathy, Science 233 (4768) (1986) 1089-1093.

[17] A. Korobeinikov, Global properties of infectious disease models with nonlinear incidence, Bulletin of Mathematical Biology 69 (6) (2007) 1871-1886.

[18] A. Korobeinikov, Global asymptotic properties of virus dynamics models with dose-dependent parasite reproduction and virulence and non-linear incidence rate, Mathematical Medicine and Biology 26 (3) (2009) 225-239.

[19] Y. Kuang, Delay differential equations: with applications in population dynamics, Academic Press, 1993.

[20] M. Y. Li, J. R. Graef, L. Wang, J. Karsai, Global dynamics of a SEIR model with varying total population size, Mathematical Biosciences 160 (2) (1999) 191-213.

[21] M. Y. Li, H. Shu, Global dynamics of an in-host viral model with intracellular delay, Bulletin of Mathematical Biology 72 (6) (2010) 1492-1505.

[22] S. P. Layne, J. L. Spouge, M. Dembo, Quantifying the infectivity of human immunodeficiency virus, Proceedings of the National Academy of Sciences 86 (12) (1989) 4644-4648.

[23] O. Lund, J. Hansen, E. Mosekilde, J. O. Nielsen, J.-E. S. Hansen, A model of enhancement and inhibition of HIV infection of monocytes by antibodies against HIV, Journal of Biological Physics 19 (2) (1993) 133-145.

[24] O. Lund, E. Mosekilde, J. Hansen, Period doubling route to chaos in a model of hiv infection of the immune system: A comment on the anderson-may model, Simulation Practice and Theory 1 (2) (1993) 49-55.

[25] C. C. McCluskey, Complete global stability for an SIR epidemic model with delay-distributed or discrete, Nonlinear Analysis: Real World Applications 11 (1) (2010) 55-59. 
[26] A. McLean, T. Kirkwood, A model of human immunodeficiency virus infection in T helper cell clones, Journal of Theoretical Biology 147 (2) (1990) 177-203.

[27] J. E. Mittler, B. Sulzer, A. U. Neumann, A. S. Perelson, Influence of delayed viral production on viral dynamics in HIV-1 infected patients, Mathematical Biosciences 152 (2) (1998) 143-163.

[28] Y. Nakata, Global dynamics of a cell mediated immunity in viral infection models with distributed delays, Journal of Mathematical Analysis and Applications 375 (1) (2011) 14-27.

[29] P. W. Nelson, J. D. Murray, A. S. Perelson, A model of HIV-1 pathogenesis that includes an intracellular delay, Mathematical Biosciences 163 (2) (2000) $201-215$.

[30] P. W. Nelson, A. S. Perelson, Mathematical analysis of delay differential equation models of HIV-1 infection, Mathematical Biosciences 179 (1) (2002) $73-94$.

[31] M. A. Nowak, R. M. Anderson, A. R. McLean, T. Wolfs, J. Goudsmit, R. M. May, Antigenic diversity thresholds and the development of aids, Science 254 (5034) (1991) 963-969.

[32] J. M. Orenstein, C. Fox, S. M. Wahl, Macrophages as a source of HIV during opportunistic infections, Science 276 (5320) (1997) 1857-1861.

[33] K.A. Pawelek, S. Liu, F. Pahlevani, L. Rong, A model of HIV-1 infection with two time delays: mathematical analysis and comparison with patient data, Mathematical Bioscience 235 (2012) 98-109.

[34] A. S. Perelson, P. W. Nelson, Mathematical analysis of HIV-1 dynamics in vivo, SIAM Review 41 (1) (1999) 3-44.

[35] M. Pope, M.G.H. Betjes, N. Romani, H. Hirmand, P.U. Cameron, L. Hoffman, S. Gezelter, G. Schuler, R.M. Steinman, Conjugates of dendritic cells and memory T lymphocytes from skin facilitate productive infection with HIV-1, Cell 78 (3) (1994) 389-398.

[36] G. Reibnegger, D. Fuchs, A. Hausen, E. R. Werner, M. P. Dierich, H. Wachter, Theoretical implications of cellular immune reactions against helper lymphocytes infected by an immune system retrovirus, Proceedings of the National Academy of Sciences 84 (20) (1987) 7270-7274.

[37] L. Rong, Z. Feng, A.S. Perelson, Mathematical analysis of age-structured HIV-1 dynamics with combination antiretroviral therapy, SIAM Journal on Applied Mathematics 67 (2007) 731-756.

[38] L. Rong, Z. Feng, A.S. Perelson, Emergence of HIV-1 drug resistance during antiretroviral treatment, Bulletin of Mathematical Biology 69 (2007) 2027-2060.

[39] L. Rong, A. S. Perelson, Modeling HIV persistence, the latent reservoir, and viral blips, Journal of Theoretical Biology 260 (2) (2009) 308-331. 
[40] Z. F. Rosenberg, A. S. Fauci, Immunopathogenic mechanisms of HIV infection: cytokine induction of HIV expression, Immunology Today 11 (1990) 176-180.

[41] A.R. Sedaghat, J.B. Dinoso, L. Shen, C.O. Wilke, R.F. Siliciano, Decay dynamics of HIV-1 depend on the inhibited stages of the viral life cycle, Proceedings of the National Academy of Sciences 105 (2008) 4832-4837.

[42] A. Takeda, C. U. Tuazon, F. A. Ennis, Antibody-enhanced infection by HIV-1 via fc receptor-mediated entry, Science 242 (4878) (1988) 580-583.

[43] P. Van den Driessche, J. Watmough, Reproduction numbers and subthreshold endemic equilibria for compartmental models of disease transmission, Mathematical Biosciences 180 (1) (2002) 29-48.

[44] T. Wang, Z. Hu, F. Liao, W. Ma, Global stability analysis for delayed virus infection model with general incidence rate and humoral immunity, Mathematics and Computers in Simulation 89 (2013) 13-22.

[45] X. Wang, A. Elaiw, X. Song, Global properties of a delayed HIV infection model with CTL immune response, Applied Mathematics and Computation 218 (18) (2012) 9405-9414.

[46] X. Wang, S. Liu, A class of delayed viral models with saturation infection rate and immune response, Mathematical Methods in the Applied Sciences 36 (2) (2013) 125-142.

[47] X. Wang, S. Liu, X. Song, Dynamics of a non-autonomous HIV-1 infection model with delays, International Journal of Biomathematics 6 (5) (2013) 1-26.

[48] X. Wang, Y. Tao, X. Song, A delayed HIV-1 infection model with BeddingtonDeangelis functional response, Nonlinear Dynamics 62 (1-2) (2010) 67-72.

[49] X. Wang, Y. Tao, X. Song, Global stability of a virus dynamics model with Beddington-Deangelis incidence rate and CTL immune response, Nonlinear Dynamics 66 (4) (2011) 825-830.

[50] M. Zöller, D. Lopatta, B. Benato, G. Andrighetto, Oscillation of antibody production and regulatory $\mathrm{T}$ cells in response to antigenic stimulation, European Journal of Immunology 15 (12) (1985) 1198-1203. 


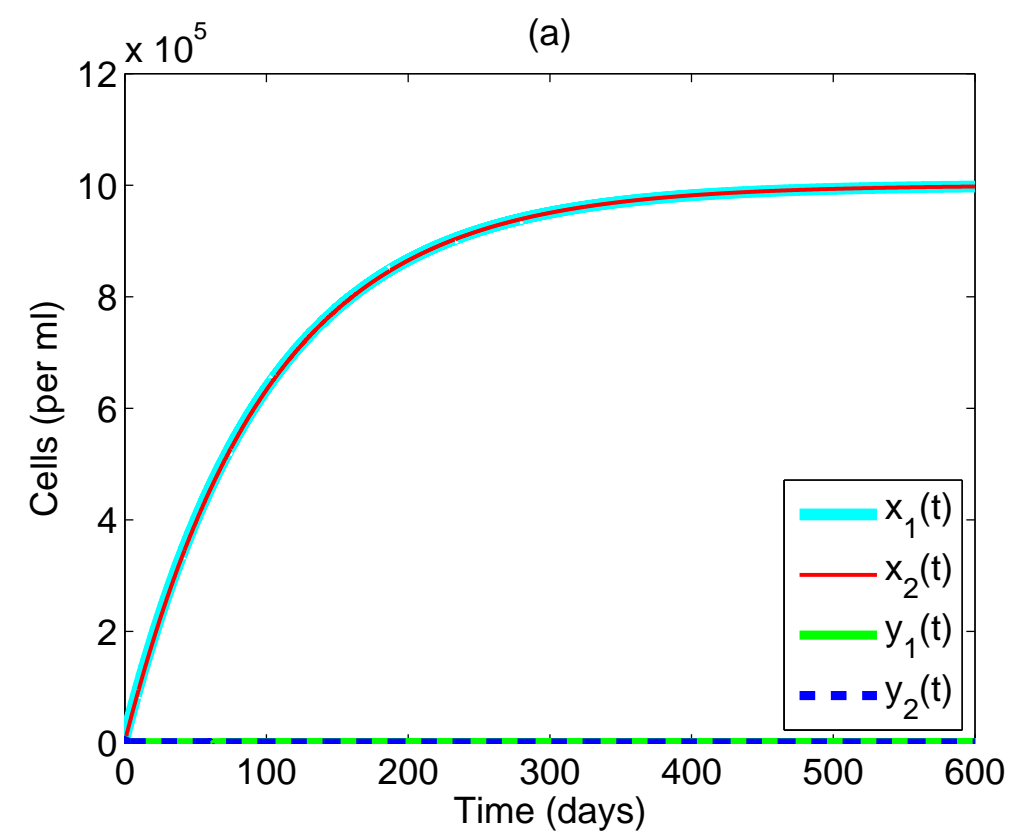

(b)

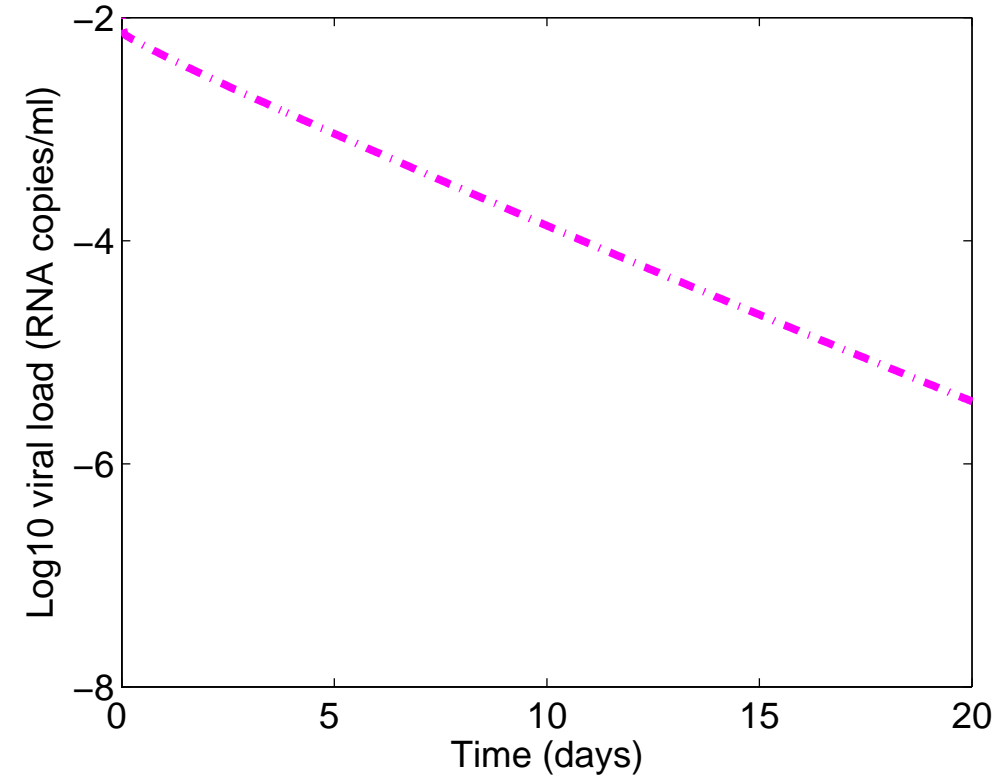

Fig. 1. Dynamics of system (2) with $\varphi_{i}\left(x_{i}(t), v(t)\right)=\beta_{i} x_{i}(t) v(t)$ and $G_{i}\left(y_{i}\right)=y_{i}$. The basic reproductive number is $R_{0} \approx 0.361<1$. The virus is predicted to be cleared and uninfected cells increase to the pre-infection level. 


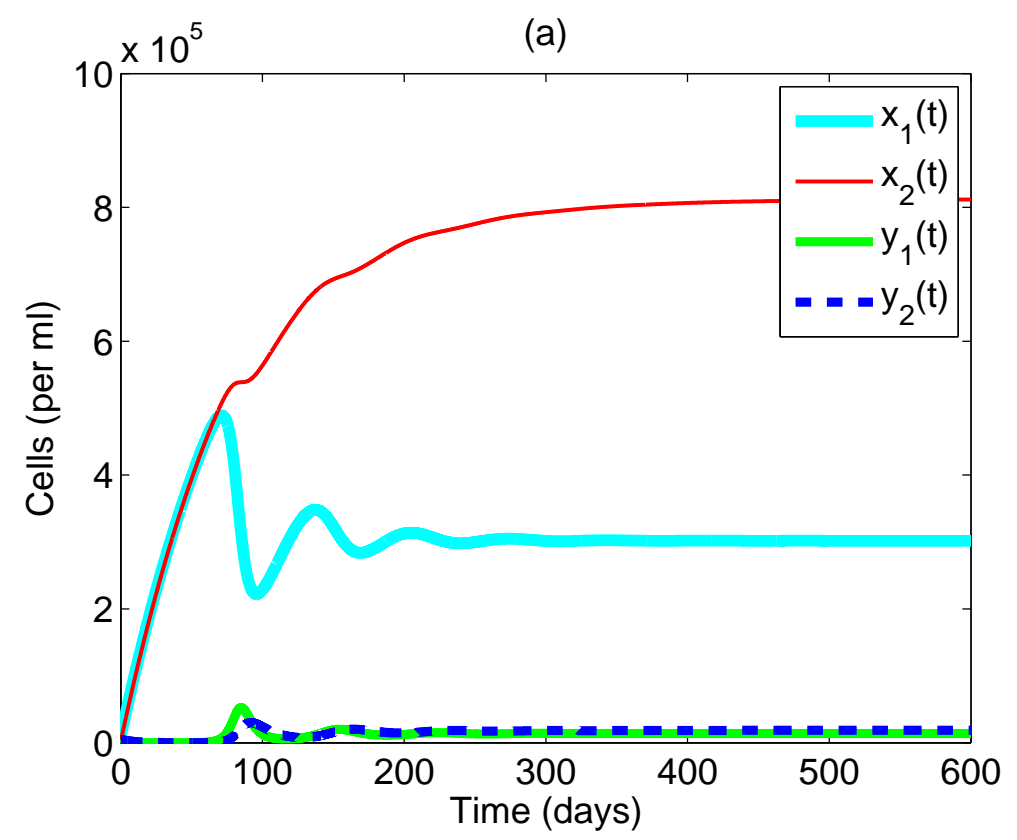

(b)

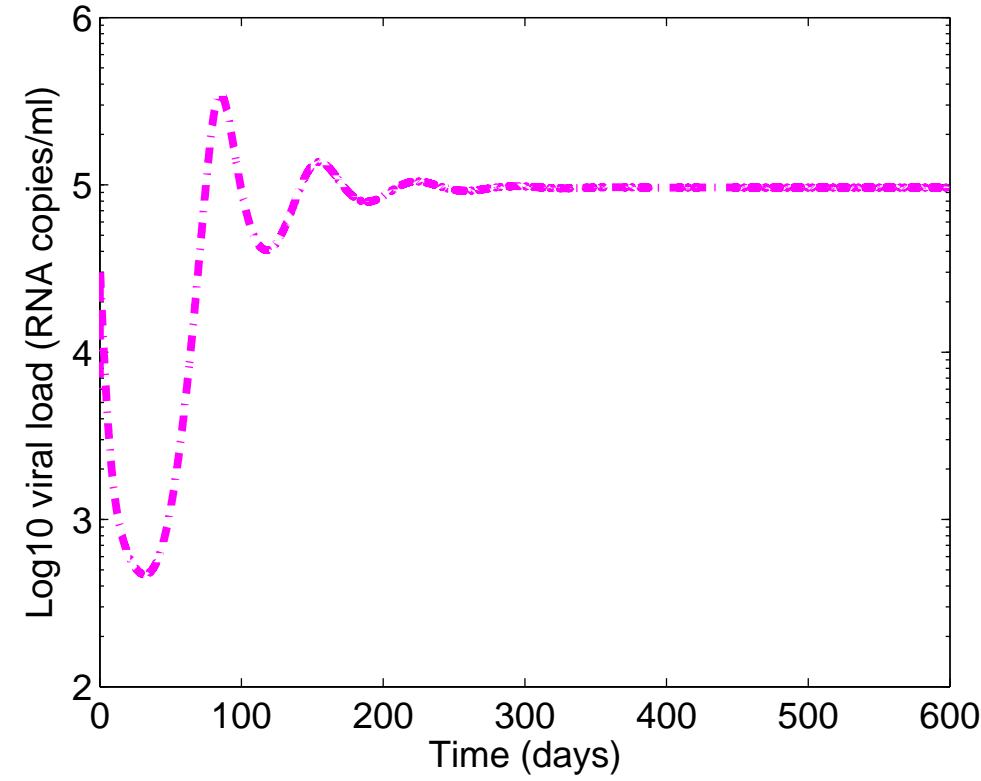

Fig. 2. Dynamics of system (2) with $\varphi_{i}\left(x_{i}(t), v(t)\right)=\beta_{i} x_{i}(t) v(t)$ and $G_{i}\left(y_{i}\right)=y_{i}$. The basic reproductive number is $R_{0} \approx 3.17>1$. Viral infection is established and the viral load converges to a steady state. 
(a)

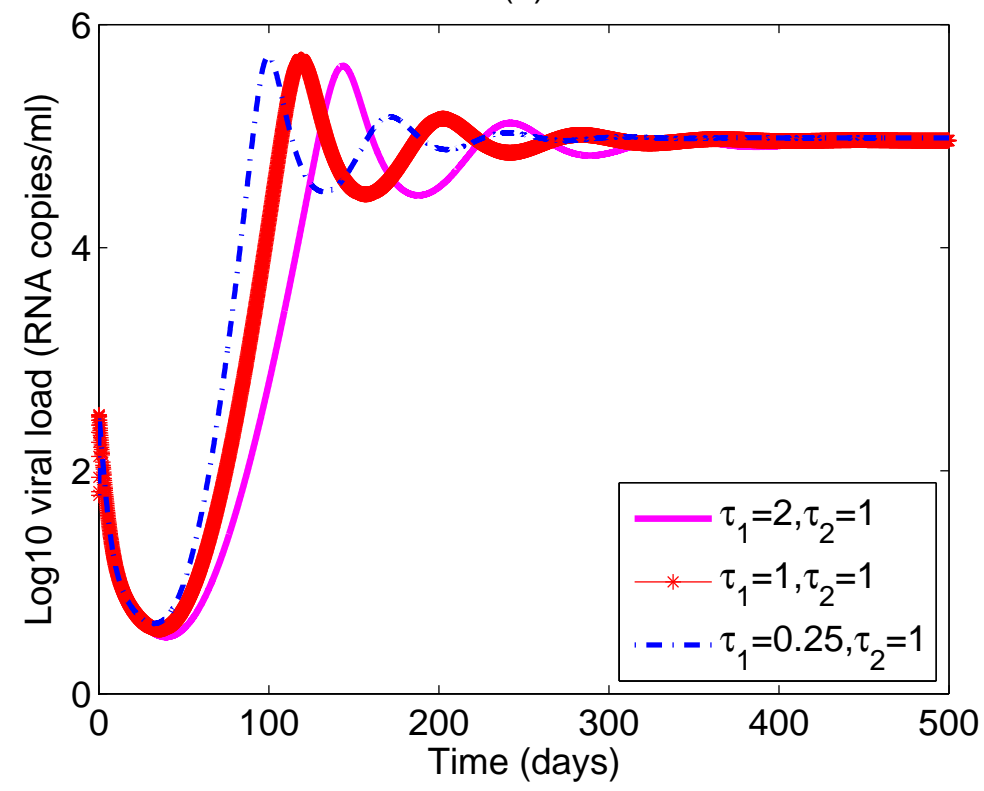

(b)

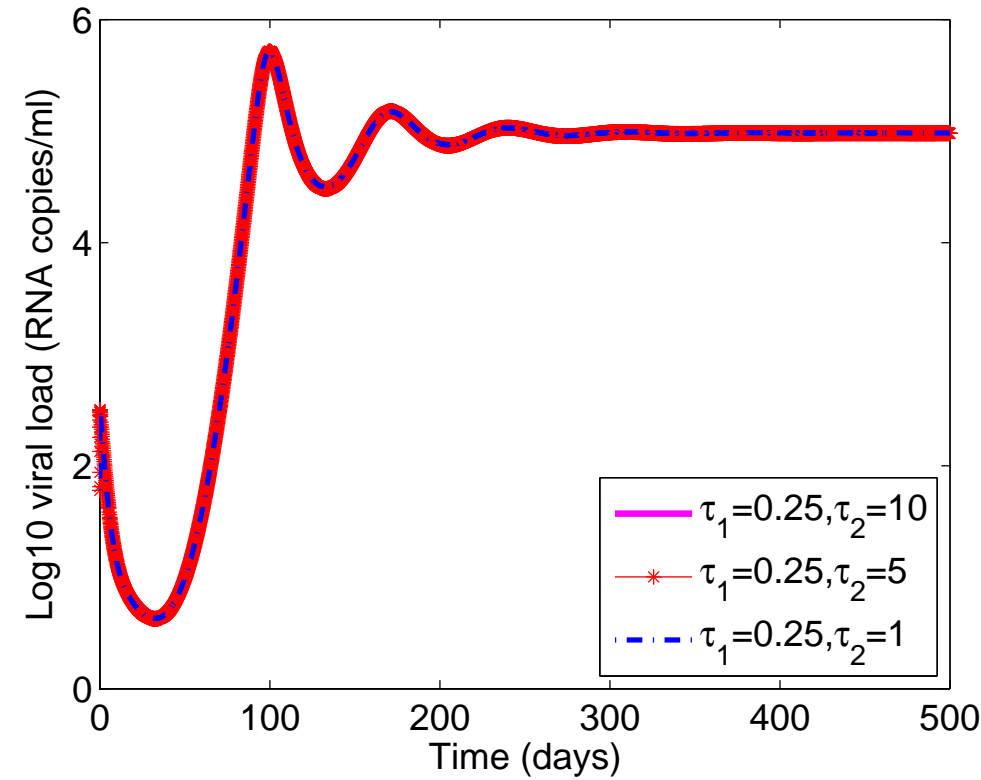

Fig. 3. Effect of time delays on the viral load change in model (2). (a) $\tau_{2}$ is fixed at 1 day and $\tau_{1}$ increases from 0.25 to 2 days. (b) $\tau_{1}$ is fixed at 0.25 days and $\tau_{2}$ increases from 1 to 10 days. Other parameters are the same as those in Fig. 2. 
(a)

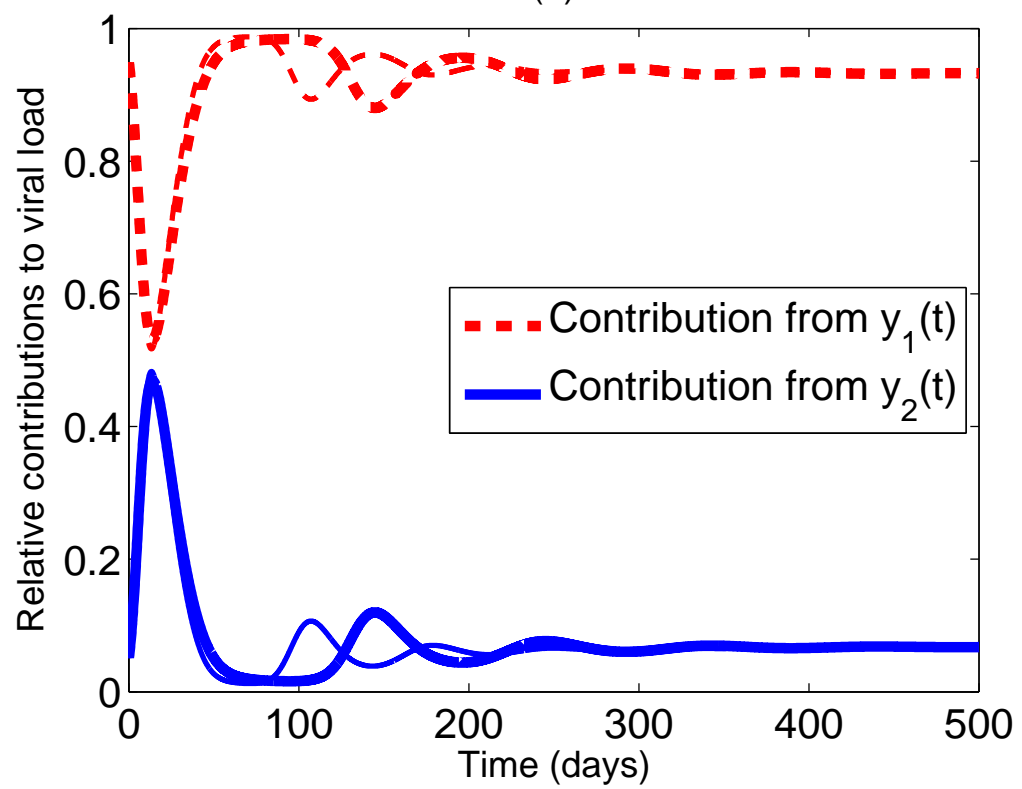

(b)

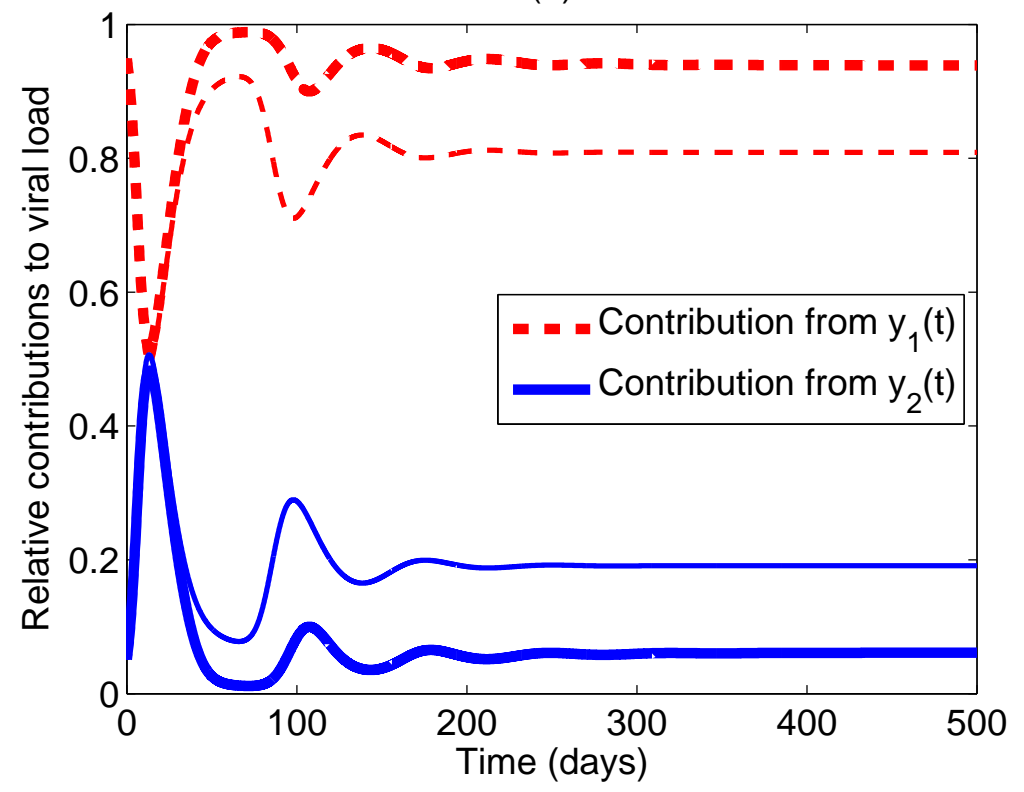

Fig. 4. The relative contribution to viral production from infected CD4+ T cells and macrophages. (a) $\tau_{2}=1$ day is fixed and $\tau_{1}$ increases from 0.25 to 2 days (the thick line represents the case of $\tau_{1}=2$ days). (b) $\tau_{1}=0.25$ days is fixed and $\tau_{2}$ increases from 0.5 to 5 days (the thick line is the case of $\tau_{2}=5$ days). Other parameters used are the same as those in Fig. 2. 